\title{
Higher-dimensional numerical relativity: \\ Formulation and code tests
}

\author{
Hirotaka Yoshino \\ Department of Physics, University of Alberta, \\ Edmonton, Alberta, Canada T6G $2 G^{r} 7$ \\ Masaru Shibata \\ Yukawa Institute for Theoretical Physics, \\ Kyoto University, Kyoto, 606-8502, Japan
}

(Dated: July 16, 2009)

\begin{abstract}
We derive a formalism of numerical relativity for higher-dimensional spacetimes and develop numerical codes for simulating a wide variety of five-dimensional (5D) spacetimes for the first time. First, the Baumgarte-Shapiro-Shibata-Nakamura formalism is extended for arbitrary spacetime dimensions $D \geq 4$, and then, the so-called cartoon method, which was originally proposed as a robust method for simulating axisymmetric $4 \mathrm{D}$ spacetimes, is described for $5 \mathrm{D}$ spacetimes of several types of symmetries. Implementing 5D numerical relativity codes with the cartoon methods, we perform test simulations by evolving a 5D Schwarzschild spacetime and a 5D spacetime composed of a gravitational-wave packet of small amplitude. The numerical simulations are stably performed for a sufficiently long time, as done in the $4 \mathrm{D}$ case, and the obtained numerical results agree well with the analytic solutions: The numerical solutions are shown to converge at the correct order. We also confirm that a longterm accurate evolution of the 5D Schwarzschild spacetime is feasible using the so-called puncture approach. In addition, we derive the Landau-Lifshitz pseudotensor in arbitrary dimensions, and show that it gives a robust tool for computing the energy flux of gravitational waves. The formulations and methods developed in this paper provide a powerful tool for studying nonlinear dynamics of higher-dimensional gravity.

PACS numbers: 04.25.D-, 04.50.-h
\end{abstract}




\section{INTRODUCTION}

Clarifying the nature of higher-dimensional gravity has become an important issue, since the braneworld scenarios were proposed [1, 2]. If the space in which we live is a threedimensional (3D) brane in extra spatial dimensions that are large or warped, the Planck energy may be of $O(\mathrm{TeV})$ and quantum gravity phenomena may emerge in high-energy particle colliders such as the LHC. If this scenario is correct, mini black holes may be produced at the LHC [3, 4, 5], and this fact motivated a lot of theoretical works in the past decade (see [6] for a recent review). Understanding the AdS/CFT correspondence is also an interesting issue in the higher-dimensional gravity.

To study nonlinear dynamics of spacetimes, numerical relativity is probably the unique approach. In the past decade, numerical relativity for four-dimensional (4D) spacetimes was significantly developed. Now, it is feasible to perform a longterm simulation for merger of binary black holes or for high-velocity collision of two black holes, which is one of the strongest gravitational phenomena in nature (see Refs. [7, 8, 9, 10, 11, 12] for pioneer works of binary black hole merger). It is natural to expect that the formulation and numerical techniques developed for 4D cases can be extended to the higher-dimensional cases.

There are also a few pioneer works in the five-dimensional (5D) numerical relativity performed in the past decade [13, 14]. However, the purpose of these works was to study a specific issue, i.e., the Gregory-Laflamme instability of a black string. Thus, the formulation and numerical method in these works are applicable only for this particular issue, and thus, developing a general formulation and codes in the higher-dimensional numerical relativity is still an issue. Furthermore, there obviously remain a lot of interesting issues to be explored in this field, as partially listed in the following.

The first issue is black hole formation in high-energy particle collisions. If a black hole is formed at the LHC, it will emit the Hawking radiation and may be detected. To predict the rate of mini black hole production and its detectability, it is necessary to know the cross section for the black hole production $\sigma_{\mathrm{BH}}$ and the resulting mass and angular momentum of the formed black hole. A partial answer was given in Refs. [15, 16] (see also [17]) by numerically solving the apparent horizon at an instant of the collision of Aichelburg-Sexl particles [18] in higher dimensions. Because the apparent horizon formation implies the formation of the event horizon assuming the cosmic censorship (e.g., see [19]), the cross 
section of the apparent horizon formation $\sigma_{\mathrm{AH}}$ gives the lower bound of $\sigma_{\mathrm{BH}}$. However, the precise value of $\sigma_{\mathrm{BH}}$ itself is necessary for exactly predicting the phenomena in the particle collider. In the 4D case, high-velocity collisions of two relativistic objects have been studied by full numerical relativity, via a model of high-velocity collision of two black holes 20, 21, 22]. In particular, Ref. [21] (see also Ref. [22] for the refinement of the work of [21]) first studied the collisions with nonzero impact parameters and clarified that $\sigma_{\mathrm{BH}}$ is approximately twice as large as $\sigma_{\mathrm{AH}}$ found in Ref. [16]. They also showed that resulting mass and angular momentum should be significantly smaller than the initial values of the system because of a huge amount of gravitational radiation. However, these studies are nothing but a prelude of the study of high-velocity collisions in higher-dimensional spacetimes, which is really required.

The second issue is on the stability of higher-dimensional rotating black holes (MyersPerry black hole) [23]. Although there are works on the stability of those black holes by separating variables for the metric perturbation in the linearized Einstein equation, the analysis can be applied for limited situations (see, e.g., [24] for special rotation parameters and [25] for a tensor-mode perturbation) and the problem has not been entirely investigated. Hence, multidimensional numerical analyses are required. Clarifying the stability of the higher-dimensional rotating black holes with a single rotation parameter, $a$, is important, because such a black hole would be the outcome of particle collisions in the TeV gravity scenarios unless the formed black hole is unstable. However, a recent numerical analysis of the linearized Einsteins equation suggests that black holes of high values of $a$ are unstable at least for spacetime dimensions $D \geq 7$ [26] (see also [27]): The production rate of mini black holes may be smaller than what we naively expect from the analysis of the apparent horizon [16]. To elucidate the stability and subsequent evolution after the onset of instability, numerical relativity will play a crucial role.

The third issue is on the evolution of a black hole on a Randall-Sundrum (RS) brane. So far, no analytic solution of a 5D static black hole localized on the RS brane has been found. The recent numerical work [28] (see also [29]) indicates the nonexistence of such solutions. If this is the case, any black hole produced on the RS brane cannot relax to a stationary state but evolve in time. Clarifying the fate of such black holes is an interesting issue. Furthermore, if the AdS/CFT correspondence holds for the RS models, a 5D classical black hole on the RS brane is dual to a $4 \mathrm{D}$ black hole with quantum fields [30, 31], and thus 
we could obtain an indication for the Hawking radiation including the back-reaction effects.

Motivated by these issues, we have developed numerical relativity codes for simulating $5 \mathrm{D}$ spacetimes as the first step. The purposes of this paper are the following three. The first purpose is to describe a numerical relativity formulation in higher-dimensional spacetimes. We adopt the Baumgarte-Shapiro-Shibata-Nakamura (BSSN) formalism [32, 33] and write down its higher-dimensional version.

The second purpose is to describe the cartoon method [34, 35] for 5D spacetimes of several types of symmetries. The cartoon method was originally proposed for simulating 4D spacetimes of axial symmetry using the Cartesian coordinates. This method has been demonstrated to be quite robust for accurately and stably simulating not only vacuum spacetimes but also rotating stars and rotating stellar core collapses (e.g., Ref. [36]). The essence of this method is that we do not have to use curvilinear coordinates that have coordinate singularities. In most higher-dimensional problems, the spacetime should have symmetries, e.g., among the extra-dimensional directions. For such problems, it will be better not to adopt the curvilinear coordinates but to adopt the Cartesian coordinates for an accurate and stable simulation, as we have learned in the 4D simulations. In the higherdimensional issues that we listed above, several types of symmetries may be imposed. For example, in the off-axis collision of two black holes, the axes perpendicular to the orbital plane should be equivalent. In this paper, we particularly focus on 4D spaces (i.e., 5D spacetimes) with $U(1)$ symmetry, $U(1) \times U(1)$ symmetry, and $S O(3)$ symmetry.

The third purpose is to report our new codes for simulating 5D spacetimes, which are implemented using the BSSN formalism and cartoon methods. For demonstrating that the codes work well, we perform simulations for test problems for which analytical solutions are known. Specifically, evolutions for a 5D Schwarzschild spacetime and for a 5D spacetime composed of a gravitational-wave packet of small amplitude are chosen for the tests. In the former case, we first solve the 5D Schwarzschild spacetime by our codes in the geodesic slice and show that the numerical results agree with the analytic solution derived in this paper. We also evolve the spacetime by the puncture approach [8] with the dynamical slices and $\Gamma$-driver conditions [39], and show that the longterm evolution of a black hole spacetime is feasible as in the $4 \mathrm{D}$ case. In the second test, we compare the numerical results of a gravitational-wave packet with the semianalytic solution for linearized Einstein equations given in Appendix A, and show that they agree well. In addition, we study a 
method for estimating the energy flux carried by gravitational waves. The Newman-Penrose formalism is widely used for extracting gravitational waves in the $4 \mathrm{D}$ numerical relativity. Unfortunately, such formalism has not yet been developed in higher dimensions. Here, we propose the higher-dimensional Landau-Lifshitz pseudotensor [40] for calculating the energy flux carried by gravitational waves and demonstrate that it correctly gives the amount of radiated energy.

This paper is organized as follows. In Sec. II, we derive the BSSN formalism in higher dimensions. In Sec. III, we describe the cartoon methods in 5D spacetimes of the three types of symmetries listed above. In Sec. IV, we present the numerical results of test simulations for $5 \mathrm{D}$ numerical relativity and show that they agree with the analytic solutions. We also show that the energy extraction by the Landau-Lifshitz pseudotensor works well. Section V is devoted to a summary. In Appendix A, we summarize the equations and analytic solutions for the linearized 5D Einstein equations of $U(1) \times U(1)$ symmetry or $S O(3)$ symmetry. The Landau-Lifshitz pseudotensor in higher dimensions is given in Appendix B. Throughout the paper, we use the units of $c=1$ where $c$ is the speed of light.

\section{BSSN FORMULATION IN HIGHER DIMENSIONS}

In this section, we describe the BSSN formalism [32, 33] for higher-dimensional spacetimes. After reviewing the Arnowitt-Deser-Misner (ADM) formulation for $D$ dimensions in Sec. IIA, the D-dimensional BSSN formalism is derived in Sec. IIB.

\section{A. ADM formulation}

Suppose $\mathcal{M}$ be a $D$-dimensional spacetime with a metric $g_{a b}$. Consider a sequence of $(D-1)$-dimensional spacelike hypersurfaces $\Sigma_{t}\left(\gamma_{a b}, K_{a b}\right)$ foliated by a time coordinate $t$ in $\mathcal{M}$. Here, $\gamma_{a b}$ is the induced metric of $\Sigma_{t}$ defined by $\gamma_{a b}:=g_{a b}+n_{a} n_{b}$ with the futuredirected unit normal $n_{a}$ to $\Sigma_{t}$. $K_{a b}$ is the extrinsic curvature $K_{a b}:=-(1 / 2) \mathcal{L}_{n} \gamma_{a b}$, where $\mathcal{L}_{n}$ is the Lie derivative with respect to $n^{a}$. The coordinate basis $t^{a}$ of the time coordinate $t$ is decomposed as $t^{a}=\alpha n^{a}+\beta^{a}$, where $\alpha$ is the lapse function and $\beta^{a}$ is the shift vector.

The $D$-dimensional Einstein equation ${ }^{(D)} G_{a b}=8 \pi G_{D} T_{a b}$ is decomposed into constraint and evolution equations. Here, ${ }^{(D)} G_{a b}, T_{a b}$, and $G_{D}$ are the $D$-dimensional Einstein tensor, 
the stress-energy tensor, and the gravitational constant, respectively. First, we define

$$
\rho:=T_{a b} n^{a} n^{b} ; \quad j_{a}:=-T_{b c} n^{b} \gamma_{a}^{c} ; \quad S_{a b}:=T_{c d} \gamma_{a}^{c} \gamma_{b}^{d}
$$

The Hamiltonian constraint is derived from the Gauss equation to give

$$
R+K^{2}-K_{a b} K^{a b}=16 \pi G_{D} \rho,
$$

where $R$ is the Ricci scalar of the spacelike hypersurface $\Sigma_{t}$. The momentum constraint is derived from the Codacci equation to give

$$
D_{b} K_{a}^{b}-D_{a} K=8 \pi G_{D} j_{a}
$$

where $D_{a}$ denotes the covariant derivative with respect to $\gamma_{a b}$. The evolution equation of the induced metric $\gamma_{a b}$ is derived from the definition of the extrinsic curvature as

$$
\mathcal{L}_{t} \gamma_{a b}=-2 \alpha K_{a b}+D_{a} \beta_{b}+D_{b} \beta_{a}
$$

and the evolution equation of the extrinsic curvature $K_{a b}$ is derived from the Ricci equation to give

$$
\begin{aligned}
\mathcal{L}_{t} K_{a b}=-D_{a} D_{b} \alpha+ & \alpha\left(R_{a b}-2 K_{a c} K_{b}^{c}+K_{a b} K\right) \\
& +\beta^{c} D_{c} K_{a b}+K_{c b} D_{a} \beta^{c}+K_{c a} D_{b} \beta^{c}-8 \pi G_{D} \alpha\left[S_{a b}+\frac{\rho-S}{D-2} \gamma_{a b}\right],
\end{aligned}
$$

where $R_{a b}$ denotes the Ricci tensor with respect to $\gamma_{a b}$ and $S:=S_{c}^{c}$. The $D$-dimensional equations are formally different from the $4 \mathrm{D}$ equations only in the coefficient of the last term of Eq. (5). In vacuum, the equations are independent of the value of $D$.

The above expressions are given in the covariant way. Here, we introduce coordinates $x^{i}$ that span the hypersurface $\Sigma_{t}$, where $i, j=1, \ldots, D-1$. In these coordinates, the line element is written by

$$
d s^{2}=-\alpha^{2} d t^{2}+\gamma_{i j}\left(d x^{i}+\beta^{i} d t\right)\left(d x^{j}+\beta^{j} d t\right)
$$

and the spatial components $\gamma^{i j}$ of $\gamma^{a b}$ are the inverse of $\gamma_{i j}$. The constraint and evolution equations in the coordinate expressions are obtained just by replacing the indices $a, b$ to the spatial indices $i, j$ and the Lie derivative $\mathcal{L}_{t}$ to the coordinate derivative $\partial_{t}$ in Eqs. (2), (3), (44), and (15). 


\section{B. BSSN formalism}

Now, we derive the BSSN formalism for higher-dimensional spacetimes. The basic idea of the BSSN formalism is to increase the number of variables as well as that of constraints in order to guarantee the stability in numerical computation (e.g., to kill constraint violation modes). First, $\gamma_{i j}$ is conformally transformed as

$$
\tilde{\gamma}_{i j}=\chi \gamma_{i j}
$$

where the conformal factor $\chi$ is chosen so that the determinant of $\tilde{\gamma}_{i j}$ (denoted by $\tilde{\gamma}$ ) satisfies the condition

$$
\tilde{\gamma}=1 .
$$

This is equivalent to setting $\chi=\gamma^{-1 /(D-1)}$. We choose $\tilde{\gamma}_{i j}$ and $\chi$ as the fundamental variables.

In the original BSSN formalism for the 4D spacetime, the conformal factor $e^{-4 \phi}$ was used rather than $\chi$. In the $4 \mathrm{D}$ puncture formalism, $\chi$ or $W=\chi^{1 / 2}$ is often used [8]. For evolving the puncture black holes in five dimensions, $\chi$ turned out to be a good choice. This is the reason that we choose $\chi$ as one of the fundamental variables.

Next, the extrinsic curvature is decomposed into the trace part and the trace-free part as

$$
K_{i j}=A_{i j}+\frac{K}{D-1} \gamma_{i j}
$$

where $K$ denotes the trace of $K_{i j}$ and $A_{i j}$ is the trace-free part. As in the 4D BSSN formalism, $K$ is chosen to be one of the fundamental variables. The trace-free part $A_{i j}$ is conformally transformed as

$$
\tilde{A}_{i j}:=\chi A_{i j}
$$

and $\tilde{A}_{i j}$ is chosen to be one of the fundamental variables. Hereafter, the indices of $\tilde{A}_{i j}$ and $\tilde{A}^{i j}$ are raised and lowered by the conformally transformed metric $\tilde{\gamma}^{i j}$ and $\tilde{\gamma}_{i j}$.

In terms of the variables $\chi, K, \tilde{\gamma}_{i j}$, and $\tilde{A}_{i j}$, the Hamiltonian constraint (2) and the momentum constraint (31) are rewritten as

$$
R+\frac{D-2}{D-1} K^{2}-\tilde{A}_{i j} \tilde{A}^{i j}=16 \pi G_{D} \rho,
$$

and

$$
\partial_{i} \tilde{A}^{i j}+\tilde{\Gamma}_{i k}^{j} \tilde{A}^{i k}-\frac{D-2}{D-1} \tilde{\gamma}^{i j} K_{, i}-\frac{(D-1)}{2} \frac{\chi, i}{\chi} \tilde{A}^{i j}=8 \pi G_{D} \tilde{\gamma}^{i j} j_{i}
$$


where $\tilde{\Gamma}_{i k}^{j}$ is the Christoffel symbol with respect to $\tilde{\gamma}_{i j}$ and the comma $(, i)$ denotes the derivative by $x^{i}$.

The evolution equation of $\chi$ is derived from Eq. (4) with Eqs. (7) and (8) to give

$$
\left(\partial_{t}-\beta^{i} \partial_{i}\right) \chi=\frac{2}{D-1} \chi\left(\alpha K-\partial_{i} \beta^{i}\right)
$$

Multiplying $\gamma^{a b}$ to Eq. (51) and rewriting it with Eqs. (9), (10), and (11), the evolution equation of $K$ is derived to give

$$
\left(\partial_{t}-\beta^{i} \partial_{i}\right) K=-D_{i} D^{i} \alpha+\alpha\left(\tilde{A}^{i j} \tilde{A}_{i j}+\frac{K^{2}}{D-1}\right)+\frac{8 \pi \alpha}{D-2}[(D-3) \rho+S] .
$$

Rewriting Eq. (44) with Eqs. (77), (9), (10), and (13), the evolution equation of the conformal $(D-1)$-metric is derived as

$$
\left(\partial_{t}-\beta^{k} \partial_{k}\right) \tilde{\gamma}_{i j}=-2 \alpha \tilde{A}_{i j}+\tilde{\gamma}_{i k} \partial_{j} \beta^{k}+\tilde{\gamma}_{j k} \partial_{i} \beta^{k}-\frac{2}{D-1} \partial_{k} \beta^{k} \tilde{\gamma}_{i j}
$$

The evolution equation of $\tilde{A}_{i j}$ is derived by substituting Eq. (9) with Eqs. (77) and (10) into Eq. (15) and using Eqs. (11), (13), (14), and (15), to give

$$
\begin{aligned}
\left(\partial_{t}-\beta^{k} \partial_{k}\right) \tilde{A}_{i j}=\chi[ & \left.-\left(D_{i} D_{j} \alpha\right)^{\mathrm{TF}}+\alpha\left(R_{i j}^{\mathrm{TF}}-8 \pi S_{i j}^{\mathrm{TF}}\right)\right] \\
& +\alpha\left(K \tilde{A}_{i j}-2 \tilde{A}_{i k} \tilde{A}_{j}^{k}\right)+\tilde{A}_{i k} \partial_{j} \beta^{k}+\tilde{A}_{k j} \partial_{i} \beta^{k}-\frac{2}{D-1} \partial_{k} \beta^{k} \tilde{A}_{i j},
\end{aligned}
$$

where TF denotes the trace-free part, e.g. $R_{i j}^{\mathrm{TF}}=R_{i j}-R \gamma_{i j} /(D-1)$.

The Ricci tensor is decomposed into two parts as

$$
R_{i j}=\tilde{R}_{i j}+R_{i j}^{(\chi)}
$$

where $\tilde{R}_{i j}$ is the Ricci tensor with respect to $\tilde{\gamma}_{i j}$ and $R_{i j}^{(\chi)}$ is the contribution of the conformal factor. Here, $\tilde{R}_{i j}$ has the terms $(1 / 2) \tilde{\gamma}^{k l}\left(\tilde{\gamma}_{k j, i l}+\tilde{\gamma}_{i l, k j}-\tilde{\gamma}_{k l, i j}-\tilde{\gamma}_{i j, k l}\right)$ and the first three terms could be the source of a numerical instability, as often found in $4 \mathrm{D}$ numerical relativity. For stable numerical integration, the following auxiliary variable is introduced [33]:

$$
\tilde{\Gamma}^{i}:=\gamma^{j k} \tilde{\Gamma}_{j k}^{i}=-\tilde{\gamma}_{, k}^{i k}
$$

We note that another choice $F_{i}:=\delta^{j k} \partial_{k} \tilde{\gamma}_{i j}$ can be used as well [32]. It was found that the numerical results for the test simulations in this paper do not essentially depend on the choice. 
Using the variable $\tilde{\Gamma}^{i}, \tilde{R}_{i j}$ and $R_{i j}^{(\chi)}$ are rewritten as

$$
\begin{aligned}
\tilde{R}_{i j}=-\frac{1}{2} \tilde{\gamma}^{k l} \tilde{\gamma}_{i j, k l}+\frac{1}{2}\left(\tilde{\gamma}_{k i} \partial_{j} \tilde{\Gamma}^{k}+\tilde{\gamma}_{k j} \partial_{i} \tilde{\Gamma}^{k}\right) & \\
& -\frac{1}{2}\left(\tilde{\gamma}_{i l, k} \tilde{\gamma}_{, j}^{k l}+\tilde{\gamma}_{j l, k} \tilde{\gamma}_{, i}^{k l}-\tilde{\Gamma}^{l} \tilde{\gamma}_{i j, l}\right)-\tilde{\Gamma}_{i k}^{l} \tilde{\Gamma}_{j l}^{k},
\end{aligned}
$$

and

$$
\begin{aligned}
& R_{i j}^{(\chi)}=\frac{(D-3)}{2 \chi}\left(\chi_{, i j}-\tilde{\Gamma}_{i j}^{k} \chi_{, k}\right)-\frac{(D-3)}{4} \frac{\chi_{, i} \chi, j}{\chi^{2}} \\
& \quad+\tilde{\gamma}_{i j} \tilde{\gamma}^{k l}\left[\frac{\chi, k l}{2 \chi}-(D-1) \frac{\chi_{, k} \chi, l}{4 \chi^{2}}\right]-\frac{1}{2} \tilde{\gamma}_{i j} \frac{\chi, m}{\chi} \tilde{\Gamma}^{m},
\end{aligned}
$$

where $\tilde{\gamma}=1$ is used in deriving these equations. As in the 4D case, the second derivatives of $\tilde{\gamma}_{i j}$ explicitly appear only in the first term of Eq. (19).

Since $\tilde{\Gamma}^{i}$ is one of the dynamical variables in the BSSN formalism, its evolution equation has to be derived. Substituting Eq. (15) into $\partial_{t} \tilde{\Gamma}^{i}=\partial_{j}\left(\tilde{\gamma}^{i k} \tilde{\gamma}^{j l} \tilde{\gamma}_{k l, t}\right)$ and eliminating $\tilde{A}^{i j}{ }_{j}$ with Eq. (12), we obtain

$$
\begin{aligned}
\left(\partial_{t}-\beta^{j} \partial_{j}\right) \tilde{\Gamma}^{i}=-2 \tilde{A}^{i j} \partial_{j} \alpha+2 \alpha[ & \left.\tilde{\Gamma}_{j k}^{i} \tilde{A}^{j k}-\frac{D-2}{D-1} \tilde{\gamma}^{i j} K_{, j}-8 \pi \tilde{\gamma}^{i j} j_{j}-\frac{(D-1)}{2} \frac{\chi, j}{\chi} \tilde{A}^{i j}\right] \\
& -\tilde{\Gamma}^{j} \partial_{j} \beta^{i}+\frac{2}{D-1} \tilde{\Gamma}^{i} \partial_{j} \beta^{j}+\frac{D-3}{D-1} \tilde{\gamma}^{i k} \beta_{, j k}^{j}+\tilde{\gamma}^{j k} \beta_{, j k}^{i} .
\end{aligned}
$$

In summary, the variables to be evolved are $\chi, K, \tilde{\gamma}_{i j}, \tilde{A}_{i j}$ and $\tilde{\Gamma}^{i}$ (or $F_{i}$ ), and their evolution equations are Eqs. (13), (14), (15), (16), and (21), respectively. The conditions (8) $\operatorname{tr}\left(\tilde{A}_{i j}\right)=0$, and (18) are regarded as the new constraints which arise because the dynamical variables are increased.

As shown above, the BSSN formalism for higher dimensions essentially has the same form as that for the $4 \mathrm{D}$ case, except that some coefficients are changed. Because of the change in the coefficients, the behavior of the solutions near the black hole and in the wave zone is changed. However, this change does not significantly affect the stability and accuracy in numerical computations at least for evolutions of the 5D Schwarzschild spacetime and a 5D spacetime of small-amplitude gravitational waves as shown in Sec. IV.

\section{CARTOON METHOD}

In this section, we describe the cartoon method for 5D spacetimes. The cartoon method was originally proposed as a prescription for stable numerical simulations of axisymmetric 4D 
spacetimes. The essence in this method is not to use curvilinear coordinates but to use the Cartesian coordinates [34]. We briefly review this (say, the case " $x=y, z$ ") in Sec. IIIA. Next, we extend this method to 5D spacetimes with symmetries. In higher-dimensional spacetimes, there are various types of possible symmetries. Here, we consider 4D spaces (i.e., $5 \mathrm{D}$ spacetimes) with $U(1)$ symmetry (the case " $x, y, z=w "), U(1) \times U(1)$ symmetry (the case " $x=y, z=w "$ ), and $S O(3)$ symmetry (the case " $x=y=z, w "$ ). The cartoon methods for these three cases are described in Secs. IIIB, IIIC, and IIID, respectively.

\section{A. 3D axisymmetric space}

For 3D axisymmetric spacelike hypersurfaces in a 4D spacetime, the 3D Cartesian coordinates $(x, y, z)$ can be introduced so that the vector $\partial_{\varphi}:=x \partial_{y}-y \partial_{x}$ becomes the Killing vector. In other words, each spacelike hypersurface has $U(1)$ symmetry around the $z$ axis. We refer to this case as $x=y, z$ in short.

One natural coordinate choice for this space is the cylindrical coordinates $(\rho, \varphi, z)$. If these coordinates are adopted, the problem reduces to a $2 \mathrm{D}$ problem (i.e., all quantities depend only on $\rho$ and $z$ ). However, in these coordinates, the symmetry axis $\rho=0$ is the coordinate singularity. On this coordinate singularity, one has to change the manner of finite differencing because there is no point of $\rho<0$. This sometimes (not always) causes a numerical instability, which is known as the finite discretization instability. Although it might be possible to stabilize numerical computations by appropriately modifying the finitedifferencing method, there is the case that the prescription is not simple or has not been found without numerical viscosity, e.g., issues for which a longterm simulation of rotating objects is necessary.

One can avoid this problem by using the Cartesian coordinates because they have no coordinate singularities. The shortcoming in the Cartesian coordinates is that $U(1)$ symmetry does not explicitly appear in equations, and thus, we have to solve 3D equations. Suppose that the initial data are given on the $(x, z)$ plane (i.e., $\varphi=0)$. In the case that the cylindrical coordinates are adopted, the subsequent evolution of the system is feasible with this data. However, in the Cartesian coordinates, one cannot calculate the next step only with this data, because the equations include $y$ derivatives of functions to be solved.

However, we do not have to prepare the data for all values of $y$, if the cartoon method 
is used. In this method, a few grid points in the neighborhood of the $(x, z)$ plane are prepared. The number of necessary grid points depends on the order of numerical accuracy required in the finite differencing (see the last paragraph of this subsection). Then, the data at a grid point $(x, y \neq 0, z)$ are generated using the data at a point $(\rho, 0, z)$ [i.e., on the $(x, z)$ plane], where $\rho=\sqrt{x^{2}+y^{2}}$, by use of the symmetry. Because the grid point is not located at the point $(\rho, 0, z)$ in general, the data at this point are determined by an interpolation. The method of the interpolation depends on the required order of accuracy (see the last paragraph of this subsection). Once the data at the grid points $y \neq 0$ are known, $y$ derivatives at $y=0$ are calculated and the data on the $(x, z)$ plane are evolved toward the next time step.

The symmetric relations are derived by the fact that the Lie derivative of functions with respect to the Killing vector becomes zero. For a scalar function $\Psi(x, y, z)$, the symmetric relation is

$$
\Psi(x, y, z)=\Psi(\rho, 0, z)
$$

In order to derive the symmetric relation of a contravariant vector function $T^{i}$, it is convenient to consider the coordinate transformation from the $(\rho, \varphi)$ coordinates to the Cartesian coordinates. After expressing $T^{x}(x, y, z)$ and $T^{y}(x, y, z)$ in terms of $T^{\rho}(\rho, z)$ and $T^{\varphi}(\rho, z)$, the latter two can be replaced by the relations on the $(x, z)$ plane, $T^{x}(\rho, 0, z)=T^{\rho}(\rho, z)$ and $T^{y}(\rho, 0, z)=\rho T^{\varphi}(\rho, z)$. This yields

$$
\begin{aligned}
& T^{x}(x, y, z)=(x / \rho) T^{x}(\rho, 0, z)-(y / \rho) T^{y}(\rho, 0, z), \\
& T^{y}(x, y, z)=(y / \rho) T^{x}(\rho, 0, z)+(x / \rho) T^{y}(\rho, 0, z) .
\end{aligned}
$$

The relation between $T^{z}(x, y, z)$ and $T^{z}(\rho, 0, z)$ is the same as that for a scalar, described in Eq. (22). A covariant vector $T_{i}(x, y, z)$ has the same symmetric relation as that of $T^{i}(x, y, z)$.

In a similar manner, the symmetric relation of a symmetric covariant tensor function $S_{i j}=S_{(i j)}$ is obtained. $S_{z z}(x, y, z)$ has the same relation as that for a scalar, Eq. (22), and $S_{z x}$ and $S_{z y}$ have the same relations as $x$ and $y$ components of a vector function, Eqs. (23) and (24). For the other components, the following relations are derived:

$$
\begin{aligned}
& S_{x x}(x, y, z)=(x / \rho)^{2} S_{x x}(\rho, 0, z)+(y / \rho)^{2} S_{y y}(\rho, 0, z)-\left(2 x y / \rho^{2}\right) S_{x y}(\rho, 0, z), \\
& S_{y y}(x, y, z)=(y / \rho)^{2} S_{x x}(\rho, 0, z)+(x / \rho)^{2} S_{y y}(\rho, 0, z)+\left(2 x y / \rho^{2}\right) S_{x y}(\rho, 0, z), \\
& S_{x y}(x, y, z)=\left(x y / \rho^{2}\right)\left[S_{x x}(\rho, 0, z)-S_{y y}(\rho, 0, z)\right]+\left[\left(x^{2}-y^{2}\right) / \rho^{2}\right] S_{x y}(\rho, 0, z) .
\end{aligned}
$$


Again, a contravariant symmetric tensor $S^{i j}$ has the same symmetric relation as that of $S_{i j}$.

Using the above relations, the data for $y \neq 0$ are generated using the data in the $(x, z)$ plane, and thus, the derivatives with respect to $y$ can be calculated. The required grid number is 5 for the fourth-order finite differencing (i.e., the data at $y= \pm \Delta y$ and $\pm 2 \Delta y$ have to be determined, where $\Delta y$ is the grid spacing), and 3 for the second-order one. For obtaining the values at a point $(\rho, z)$ on the $(x, z)$ plane, interpolation is necessary. To keep the fourth-order accuracy, we have to use at least fourth-order accurate interpolation (e.g., fourth-order Lagrangian interpolation).

\section{B. 4D space with $U(1)$ symmetry}

In the following, we describe three cartoon methods in $5 \mathrm{D}$ spacetimes (4D spaces) of three types of symmetries, denoting the Cartesian coordinates by $(x, y, z, w)$ and assuming that the 4D space is topologically identical to the 4D Euclidean space.

First, we consider a $4 \mathrm{D}$ space of $U(1)$ symmetry whose corresponding Killing vector is $\partial_{\psi}=z \partial_{w}-w \partial_{z}$ (i.e., $\left.\tan \psi=w / z\right)$. An example of a system of this symmetry is an off-axis collision of two black holes. Suppose that the centers of the two black holes are located in the $(x, y)$ plane. In this case, the directions orthogonal to the $(x, y)$ plane (i.e., the direction of $z$ and $w$ axes) are equivalent, and thus, the space has the $U(1)$ symmetry. We refer to this symmetric space as $x, y, z=w$ in short.

Such a spacetime can be simulated as a $3+1$ problem using the cartoon method in a similar prescription to that in the 3D axisymmetric space. We first prepare the grid points in the $(x, y, z)$ plane and a few neighboring grid points in the $w$ direction. Then, the data at a point $(x, y, z, w \neq 0)$ are generated by the data at a point $(x, y, \rho, 0)$ with symmetric relations, where $\rho=\sqrt{z^{2}+w^{2}}$. The symmetric relations are essentially same as those in the 3D axisymmetric case: It is sufficient to replace the indices $(x, y)$ in Eqs. (23)-(27) to $(w, z)$. As for the other components, $T^{x}, T^{y}, S_{x x}, S_{y y}$, and $S_{x y}$ behave like scalar functions, and $\left(S_{x z}, S_{x w}\right)$ and $\left(S_{y z}, S_{y w}\right)$ behave like $z$ and $w$ components of vector functions $\left(T^{z}, T^{w}\right)$. 


\section{4 D space with $U(1) \times U(1)$ symmetry}

Next, we describe the cartoon method in a $4 \mathrm{D}$ space of $U(1) \times U(1)$ symmetry, where two Killing vector fields, $\partial_{\varphi}=x \partial_{y}-y \partial_{x}$ and $\partial_{\psi}=z \partial_{w}-w \partial_{z}$, are present. An example of a spacetime of this symmetry is a $5 \mathrm{D}$ rotating black hole spacetime with two rotation parameters [23]. In this spacetime, the black hole is rotating with respect to the $(x, y)$ and $(z, w)$ planes simultaneously. Of course, a 5D rotating black hole with one rotation parameter is also the case for this symmetry. We refer to such a case as " $x=y, z=w$ " in short.

The spacetime of this symmetry can be simulated as a $2+1$ problem in the cartoon method. We prepare grid points on the $(x, z)$ plane and a few neighboring grid points in both $y$ and $w$ directions. In this symmetry, two cartoons are necessary: The first cartoon to generate the data in the $y$ - direction, and the second cartoon to generate the data in the $w$ - direction. The symmetric relations for each cartoon are essentially the same as those in the previous two subsections.

In numerical simulation, we have the data of points $(x, 0, z, 0)$ at each time step. Then, we apply the first cartoon to generate the data for grid points $(x, y, z, 0)$. After that, we apply the second cartoon to generate the data for grid points $(x, y, z, w)$. Then, all the necessary derivatives with respect to $y$ and $w$ can be taken and the data can be evolved to the next time step. This method may be called the double cartoon method. As we demonstrate in Sec. IV, the double cartoon method works well as the single cartoon method.

\section{4D space with $S O(3)$ symmetry}

Finally, we consider a space of a different type of symmetry, $S O(3)$ symmetry, in which the three Killing vectors, $\boldsymbol{\xi}_{1}:=y \partial_{z}-z \partial_{y}, \boldsymbol{\xi}_{2}:=z \partial_{x}-x \partial_{z}$, and $\boldsymbol{\xi}_{3}:=x \partial_{y}-y \partial_{x}$ are present. In other words, each hypersurface of $w=$ const. is spherically symmetric. An example for a spacetime of this symmetry is a head-on collision of two black holes moving along the $w$ axis, because the other directions $x, y$, and $z$ are equivalent if the black holes are not rotating. We refer to this case as $x=y=z, w$ in short.

This spacetime can be simulated as a $2+1$ problem in the cartoon method. We prepare grid points in the $(x, w)$ plane and a few neighboring grid points in both $y$ and $z$ directions. 
Using the data on the $(x, w)$ plane, the data at points $(x, y, z, w)$ can be calculated by the $S O(3)$ symmetric relations using the data at the point $(r, 0,0, w)$ where $r=\sqrt{x^{2}+y^{2}+z^{2}}$. The data at the point $(r, 0,0, w)$ should be determined by an interpolation as before.

In this case, the symmetry relations are different from those in the previous cases. For scalar functions, it is trivial as

$$
\Psi(x, y, z, w)=\Psi(r, 0,0, w)
$$

In order to derive the symmetry relations for vector and symmetric tensor functions, we have to know the $S O(3)$-symmetric forms of a vector and a symmetric tensor, which can be found by the conditions $\mathcal{L}_{\boldsymbol{\xi}_{n}} T^{i}=0$ and $\mathcal{L}_{\boldsymbol{\xi}_{n}} S_{i j}=0$. For this purpose, we first describe their components in the spherical-polar coordinates $(r, \theta, \varphi, w)$ introduced by $x=r \sin \theta \cos \varphi$, $y=r \sin \theta \sin \varphi$, and $z=r \cos \theta$. Then, in the $S O(3)$ symmetry, they are written as

$$
T^{i}(r, \theta, \varphi, w)=\left(T^{r}(r, w), 0,0, T^{w}(r, w)\right),
$$

and

$$
S_{i j}(r, \theta, \varphi, w)=\left(\begin{array}{cccc}
S_{r r}(r, w) & 0 & 0 & S_{r w}(r, w) \\
* & S_{\theta \theta}(r, w) & 0 & 0 \\
* & * & S_{\theta \theta}(r, w) \sin ^{2} \theta & 0 \\
* & * & * & S_{w w}(r, w)
\end{array}\right) .
$$

Now we transform these expressions to the Cartesian coordinates and use the relations on the $(x, w)$ plane to give

$$
\begin{aligned}
& T^{x}(x, y, z, w)=(x / r) T^{x}(r, 0,0, w), \\
& T^{y}(x, y, z, w)=(y / r) T^{x}(r, 0,0, w), \\
& T^{z}(x, y, z, w)=(z / r) T^{x}(r, 0,0, w),
\end{aligned}
$$

for a vector function and

$$
\begin{gathered}
S_{x x}(x, y, z, w)=\left(x^{2} / r^{2}\right) S_{x x}(r, 0,0, w)+\left(1-x^{2} / r^{2}\right) S_{y y}(r, 0,0, w), \\
S_{y y}(x, y, z, w)=\left(y^{2} / r^{2}\right) S_{x x}(r, 0,0, w)+\left(1-y^{2} / r^{2}\right) S_{y y}(r, 0,0, w), \\
S_{z z}(x, y, z, w)=\left(z^{2} / r^{2}\right) S_{x x}(r, 0,0, w)+\left(1-z^{2} / r^{2}\right) S_{y y}(r, 0,0, w), \\
S_{y z}(x, y, z, w)=\left(y z / r^{2}\right)\left[S_{x x}-S_{y y}\right](r, 0,0, w), \\
S_{z x}(x, y, z, w)=\left(z x / r^{2}\right)\left[S_{x x}-S_{y y}\right](r, 0,0, w), \\
S_{x y}(x, y, z, w)=\left(x y / r^{2}\right)\left[S_{x x}-S_{y y}\right](r, 0,0, w),
\end{gathered}
$$


for a tensor function. Note that $T^{w}$ and $S_{w w}$ satisfy the symmetry relation of a scalar function, and $\left(S_{w x}, S_{w y}, S_{w z}\right)$ satisfy that of $(x, y, z)$ components of a vector function.

Using these symmetry relations, one can calculate the data at grid points in the neighborhood of the $(x, w)$ plane (i.e., $y, z \neq 0)$, and thus the evolution can be performed as a $2+1$ problem. Note that by eliminating the $w$ direction, the above symmetry relations can be used also for simulating a $3 \mathrm{D}$ spherically symmetric space in a $4 \mathrm{D}$ spacetime.

\section{CODE TESTS}

In the previous two sections, we have described necessary ingredients for higherdimensional numerical relativity, i.e., the BSSN formalism and the cartoon method. Based on these, we have implemented several codes for simulating $5 \mathrm{D}$ spacetimes in the following manner. As often done in the 4D numerical relativity (e.g., Ref. [37]), we adopt the centered fourth-order finite differencing in the space directions, except the advection terms such as $\beta^{k} \partial_{k} \tilde{\gamma}_{i j}$ for which the fourth-order upwind finite differencing is adopted. The time evolution is carried out using the fourth-order Runge-Kutta method, where the Courant number is adopted to be 0.5 . Vertex-centered grids are employed for all the space directions. In the present codes, we do not implement adaptive mesh refinement (AMR) algorithm. We plan to combine our codes with our AMR code (SACRA code) in the future [38].

So far, we have developed the $3 \mathrm{D}$ codes for spacetimes with $U(1)$ symmetry $(x, y, z=w)$, the $2 \mathrm{D}$ codes for spacetimes with $U(1) \times U(1)$ symmetry $(x=y, z=w)$ and with $S O(3)$ symmetry $(x=y=z, w)$. The authors of this paper have independently developed the codes, and checked that the numerical results for test simulations derived by the two codes agree. In addition, Yoshino has made a 1D code for spacetimes with $S O(4)$ symmetry ( " $x=y=z=w$ " in short). In the following, we present the results by Yoshino's code, for which the uniform grid with the grid spacing $\Delta x$ is always adopted for all the space directions.

In order to prove the validity of our codes, we consider that at least the following two test simulations have to be successfully carried out as in the $4 \mathrm{D}$ numerical relativity. One is the evolution of the 5D Schwarzschild black hole, and the other is the evolution of a spacetime composed of gravitational waves of small amplitude. Since their metrics are analytically given, they can be used in the benchmark tests for calibrating the codes. The results of 
the test simulations are reported in Secs. IVA and IVB, respectively. In addition, we show in Sec. IVB that the energy flux can be correctly calculated for linear gravitational waves using the Landau-Lifshitz pseudotensor.

\section{A. 5D Schwarzschild spacetime}

First, we analytically derive the 5D Schwarzschild metric in the geodesic slices and then compare numerical results with it. Next, we demonstrate that a long-term evolution of the 5D Schwarzschild spacetime is feasible in the so-called puncture approach, as in the 4D case (e.g., Refs. [8, 37, 41, 42]).

\section{Geodesic slices}

The well-known metric of a 5D Schwarzschild spacetime is

$$
d s^{2}=-f(r) d t^{2}+\frac{d r^{2}}{f(r)}+r^{2} d \Omega_{3}^{2}, \quad f(r)=1-\frac{r_{h}^{2}}{r^{2}}
$$

where $d \Omega_{3}^{2}$ is the line element of a $3 \mathrm{D}$ unit sphere and $r_{h}$ is the Schwarzschild radius

$$
r_{h}=\sqrt{\frac{8 G_{5} M}{3 \pi}}
$$

Here, we consider the Gaussian normal coordinates starting from the $t=0$ hypersurface, which is analogous to the Novikov coordinates in the 4D Schwarzschild spacetime [43, 44]. Let us introduce a geodesic congruence of test particles that are initially at rest. Each geodesic labels the radial coordinate. Denoting $r_{0}$ as the initial value of $r$ for each geodesic, we define $\bar{r}$ by

$$
r_{0}=\bar{r}\left(1+\frac{r_{h}^{2}}{4 \bar{r}^{2}}\right)
$$

as the radial coordinate. At $t=0$, the coordinate $\bar{r}$ agrees with the so-called isotropic radial coordinate. Adopting the proper time $\tau$ for each geodesic as the time coordinate, the geodesic equations are solved to give

$$
r^{2}=r_{0}^{2}-\left(r_{h} / r_{0}\right)^{2} \tau^{2},
$$

and

$$
t=\sqrt{f\left(r_{0}\right)} \tau+\frac{r_{h}}{2} \log \left|\frac{\tau+\left(r_{0}^{2} / r_{h}\right) \sqrt{f\left(r_{0}\right)}}{\tau-\left(r_{0}^{2} / r_{h}\right) \sqrt{f\left(r_{0}\right)}}\right|
$$



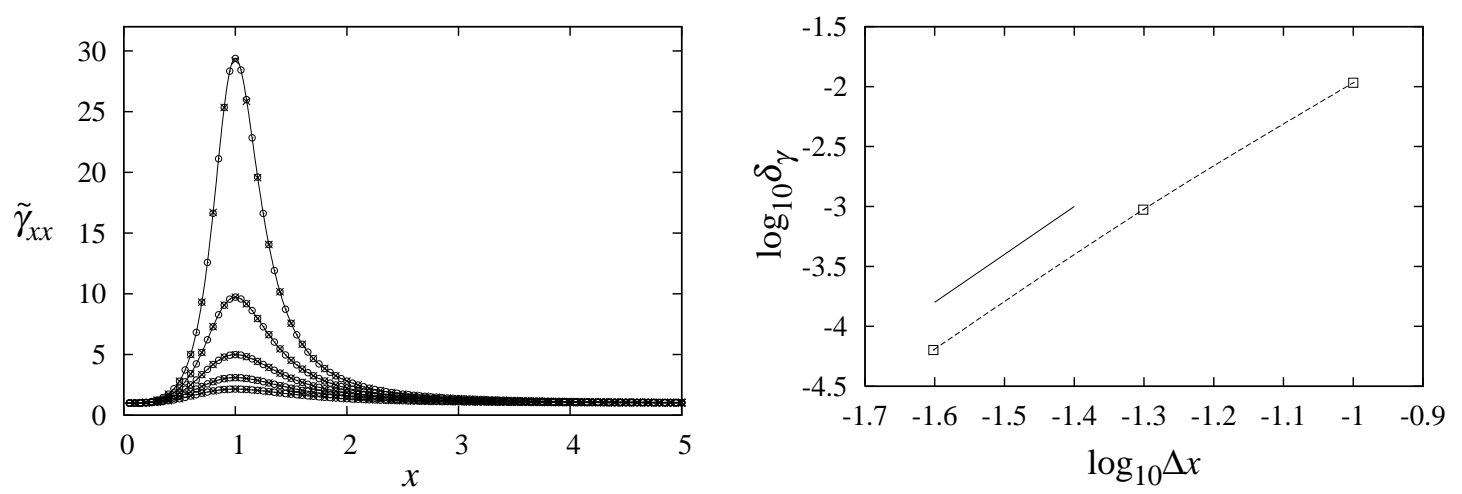

FIG. 1: Left-hand panel: Snapshots of $\tilde{\gamma}_{x x}$ along the $x$ axis for $\tau / r_{h}=0.5,0.6,0.7,0.8$, and 0.9 . The unit of $x$ is $r_{h} / 2$. The grid resolutions are $\Delta x=0.1(\times)$ and $0.05(\odot)$. The solid curves denote the analytic solutions, $\tilde{\gamma}_{x x}^{(a)}$. Right-hand panel: The averaged error, $\delta_{\gamma}$, as a function of $\Delta x$. Here, the average is taken for the data in the range $0 \leq x \leq 5$. The upper short line segment shows the relation of the fourth-order convergence (i.e., a segment with the slope 4).

Substituting these equations into Eq. (40) and transforming $r_{0}$ to $\bar{r}$ with Eq. (42), we obtain

$$
d s^{2}=-d \tau^{2}+\frac{\left[r_{0}^{2}+\left(r_{h} / r_{0}\right)^{2} \tau^{2}\right]^{2}}{\left[r_{0}^{2}-\left(r_{h} / r_{0}\right)^{2} \tau^{2}\right]} \frac{d \bar{r}^{2}}{\bar{r}^{2}}+\left[r_{0}^{2}-\left(r_{h} / r_{0}\right)^{2} \tau^{2}\right] d \Omega_{3}^{2} .
$$

This line element shows that the $\bar{r} \bar{r}$ component of the metric diverges at $\tau=r_{0}^{2} / r_{h}$. Curvature invariants indeed show that the curvature singularity appears at this time. This implies that at the time $\tau=r_{h}$, the slice hits the singularity at $\bar{r}=r_{h} / 2$.

The derived line element (45) shows the exact solution, and thus it can be used for test simulations. In this test, we perform a simulation with the gauge condition $\alpha=1$ and $\beta^{i}=0$, until the computation crashes approximately at the crash time $\tau_{\text {crash }}=r_{h}$.

The left-hand panel of Fig. 1 shows the snapshots of $x x$ component of the conformal 4D metric $\tilde{\gamma}_{x x}$ along the $x$ axis for various time slices as $\tau / r_{h}=0.5,0.6,0.7,0.8$, and 0.9 . For this plot, the grid resolutions $\Delta x=0.1$ and 0.05 are adopted. Here, the units of $x$ are $r_{h} / 2$ (i.e., the event horizon is initially located at $x=1$ ). We see that the values of $\tilde{\gamma}_{x x}$ rapidly increase and blow up around $x=1$, and agree approximately with the analytic solutions (45)) (solid curves).

The right-hand panel of Fig. 1 plots the averaged error as a function of the grid spacing $\Delta x$. Here, the averaged error is defined by

$$
\delta_{\gamma}=\frac{1}{5} \int_{0}^{5} d x\left|\tilde{\gamma}_{x x}-\tilde{\gamma}_{x x}^{(a)}\right|
$$



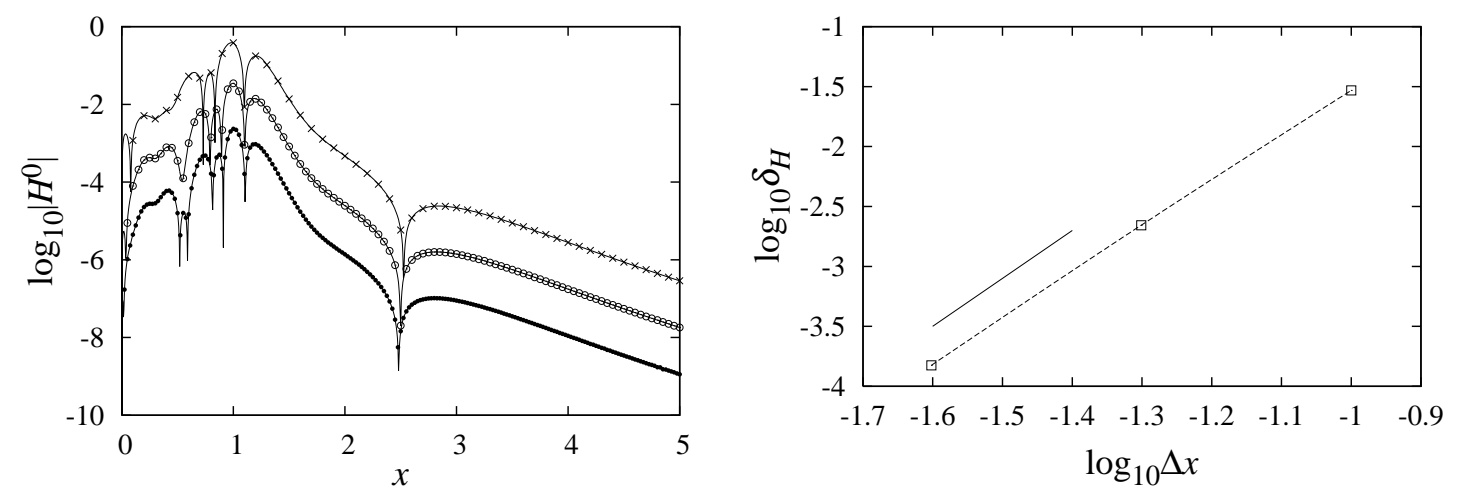

FIG. 2: Left-hand panel: The violation of the Hamiltonian constraint at the time $\tau / r_{h}=0.9$ for the grid resolutions $\Delta x=0.1(\times), 0.05(\odot)$, and $0.025(\bullet)$. Although the violation grows as the 4D hypersurface approaches the singularity, it becomes smaller for a fixed value of $\tau$ as the resolution is increased. Right-hand panel: The average, $\delta_{H}$, as a function of the grid spacing $\Delta x$. The upper short line segment shows the relation of the fourth-order convergence (i.e., a segment with the slope 4).

where $\tilde{\gamma}_{x x}$ and $\tilde{\gamma}_{x x}^{(a)}$ are numerical and analytic solutions, respectively, and the integral is numerically performed using the data on the grid points. This figure indicates that the numerical error approaches zero approximately at the fourth-order convergence.

Here, the figures are plotted for the results obtained by the 1D code $(x=y=z=w)$, but essentially the same results are obtained by the $2 \mathrm{D}$ codes $(x=y, z=w$ and $x=y=z$, $w)$ and the $3 \mathrm{D}$ code $(x, y, z=w)$.

The left-hand panel of Fig. 2 plots the violation of the Hamiltonian constraint along the $x$ axis at $\tau / r_{h}=0.9$. Here, the violation is defined by

$$
H^{0}:=R-\frac{3}{4} K^{2}+\tilde{A}_{i j} \tilde{A}^{i j}
$$

As the surface approaches the singularity, the value of the constraint violation grows rapidly. However, if we fix the time $\tau$ and compare the results by the different grid resolutions $\Delta x=0.1(\times), 0.05(\odot)$, and $0.025(\bullet)$, the clear convergence is seen.

The right-hand panel of Fig. 2 plots the averaged constraint violation $\delta_{H}$. Here, the average is defined in the same manner as Eq. (46). This figure indicates that the error converges also at the fourth order approximately. 


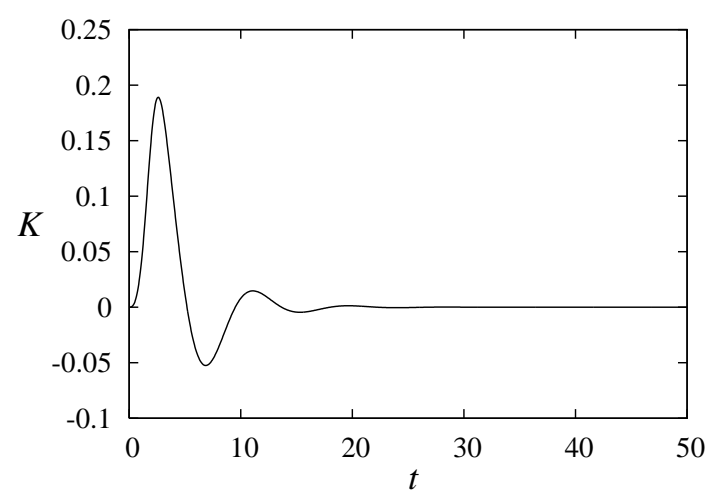

FIG. 3: The evolution of the trace of the extrinsic curvature $K$ at $x=r_{h} / 2(y=z=w=0)$ in the puncture gauge. The unit of $t$ is $r_{h} / 2$. The value of $K$ asymptotes to zero.

\section{Long-term evolution}

Next, we show that long-term evolution of a black hole is feasible using the puncture approach as in the 4D case. In this test, the initial condition is prepared in the isotropic coordinates, and then, the evolution is carried out without excising the black hole interior. As the gauge conditions, we adopt the generalized version of the dynamical slicing condition [39, 42$]$

$$
\partial_{t} \alpha=-\eta_{\alpha} \alpha K
$$

and the $\Gamma$-driver gauge condition [39]

$$
\partial_{t} \beta^{i}=\frac{(D-1)}{2(D-2)} v_{\text {long }}^{2} B^{i}, \quad \partial_{t} B^{i}=\partial_{t} \tilde{\Gamma}^{i}-\eta_{\beta} B^{i} .
$$

Here, $v_{\text {long }}$ indicates the propagation speed of a gauge mode and has to be chosen as $0<$ $v_{\text {long }} \leq 1$. We tried the choices $v_{\text {long }}=1$ and $\sqrt{3} / 2$, and found that the stable numerical evolution is possible in both cases. The choice $v_{\text {long }}=\sqrt{3} / 2$ stabilizes the numerical evolution near the puncture a little more. $\eta_{\alpha}$ and $\eta_{\beta}$ are positive constants that can be arbitrarily chosen. For $\eta_{\alpha}$, we chose several values between 1.2 and 2.0, and found that the stable and long-term simulation is feasible irrespective of the value of $\eta_{\alpha}$. For $\eta_{\beta}$, we choose $1 / 5 r_{h}$.

In the following, we show the results of the numerical evolution for the case $v_{\text {long }}=1$ and $\eta_{\alpha}=2$. The initial condition of the lapse and shift is chosen as $\alpha=\sqrt{\chi}$ and $\beta^{i}=0$. Figure 3 shows the evolution of $K$ at $x=r_{h} / 2$ on the $x$ axis. The unit of the length is $r_{h} / 2$. The value of $K$ relaxes to zero after a few oscillations, and the slice asymptotes to a maximal surface 

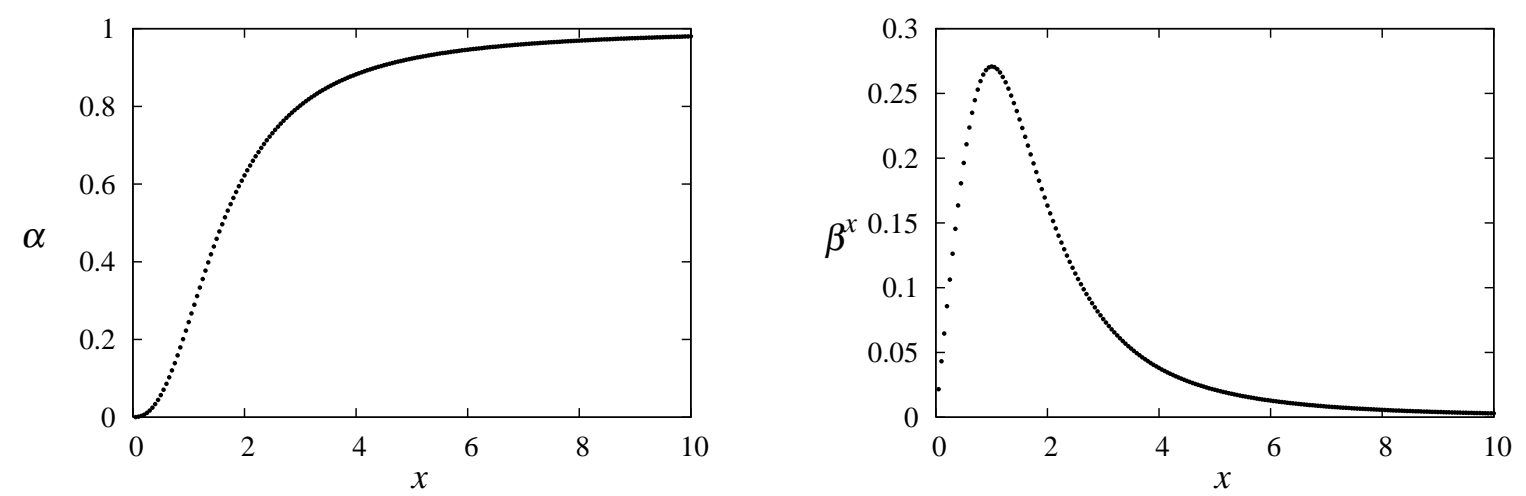

FIG. 4: The values of the lapse $\alpha$ and the shift vector $\beta^{x}$ along the $x$ axis at the time $t=50 r_{h}$. Here, the unit of $x$ is $r_{h} / 2$.

because of the property of the dynamical slicing condition (48). We evolved this spacetime up to $t=100 r_{h}$, and the spacetime relaxes to a stationary state. Figure 4 shows the values of $\alpha$ and $\beta^{x}$ along the $x$ axis at $t=50 r_{h}$. By this time, the variables approximately relax to stationary states. These results are quite similar to the evolution of a 4D Schwarzschild spacetime (compare with Figs. 1 and 5 in Ref. [42]).

The left-hand panel of Fig. 5 plots the violation from the Hamiltonian constraint defined by Eq. (47) along the $x$ axis at the time $t=50 r_{h}$ for the grid resolutions $\Delta x=0.1(\times)$, $0.05(\odot)$, and $0.025(\bullet)$. After the long-term evolution, the violation in the neighborhood of the puncture $x=0$ grows large to become $O(1)$. This is because the analyticity of the solution is violated at the puncture. However, the error rapidly decreases as $x$ is increased, indicating the reliability of the numerical results. It is also found that the spatial patterns of $H^{0}$ depend on the resolution after the longterm evolution, $t \gg r_{h}$, although initially they have similar shapes.

The right-hand panel of Fig. 5 plots the averaged constraint violation $\delta_{H}$ in the range $0.5 \leq x \leq 10$ defined in the same manner as Eq. (46). Because of the error generated at the puncture, the value of $\delta_{H}$ does not show the fourth-order convergence. Nevertheless, the violation rapidly decreases as the grid resolution is increased.

The obtained stationary data are expected to agree with those of the limit surface of the maximally sliced evolution. In the $4 \mathrm{D}$ case, the limit surface of $K=0$ was analytically determined [45, 46] and also the asymptotic solution in the numerical simulation agrees with it. A simulation [42] also demonstrates that the spacetime remains in a stationary 

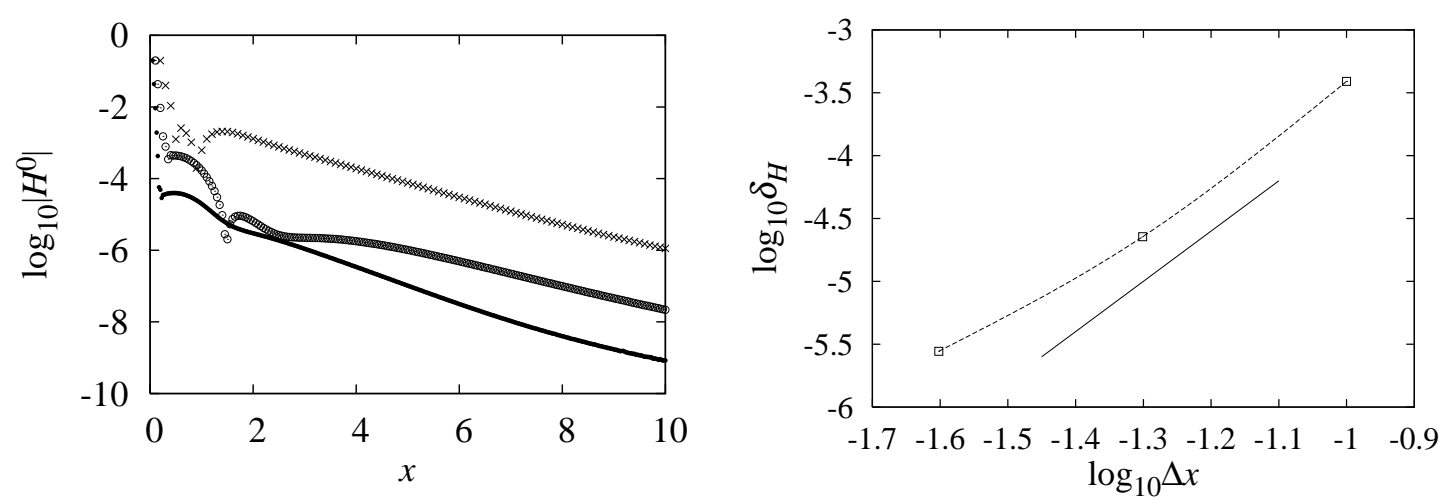

FIG. 5: Left-hand panel: The violation of the Hamiltonian constraint at the time $t=50 r_{h}$ for the grid resolutions $\Delta x=0.1(\times), 0.05(\odot)$, and $0.025(\bullet)$. After the long-term evolution, the spatial pattern of $H^{0}$ depends on the resolution, and the error generated at the puncture is $O(1)$. But the general tendency is that the violation becomes smaller as the resolution is increased. Right-hand panel: The average, $\delta_{H}$, in the range $0.5 \leq x \leq 10$ as a function of the grid spacing $\Delta x$. The lower short line segment shows the relation of the fourth-order convergence (i.e., a segment with the slope 4). The convergence is worse than the fourth-order convergence because of the error generated at the puncture.

state if the limit surface is adopted as the initial condition. The limit surface exists also in a higher-dimensional Schwarzschild spacetime and it provides a useful benchmark for calibrating codes for higher dimensions, as shown in Ref. [47].

\section{B. Linear gravitational waves}

We turn our attention to a simulation for propagation of gravitational waves of small amplitude. Here, we focus only on gravitational waves that preserve $U(1) \times U(1)$ symmetry $(x=y, z=w)$ or $S O(3)$ symmetry $(x=y=z, w)$. In Appendix A, the linearized Einstein equations of such symmetries and their special solutions for the lowest multipole moment $l$ are described. In this subsection, we pick up a tensor-mode perturbation with $U(1) \times U(1)$ symmetry for the test simulation.

As the perturbative solution used for the test simulation, we adopt the spatial metric (A35) with Eq. (A34) and the special solution for $h(t, r)$ given by Eq. (A23) with the gauge condition $\alpha=1$ and $\beta^{i}=0$. Here, we set $A=1 / 6$ and $B=0$ in Eq. (A34), and $A_{0}=0.015$ 

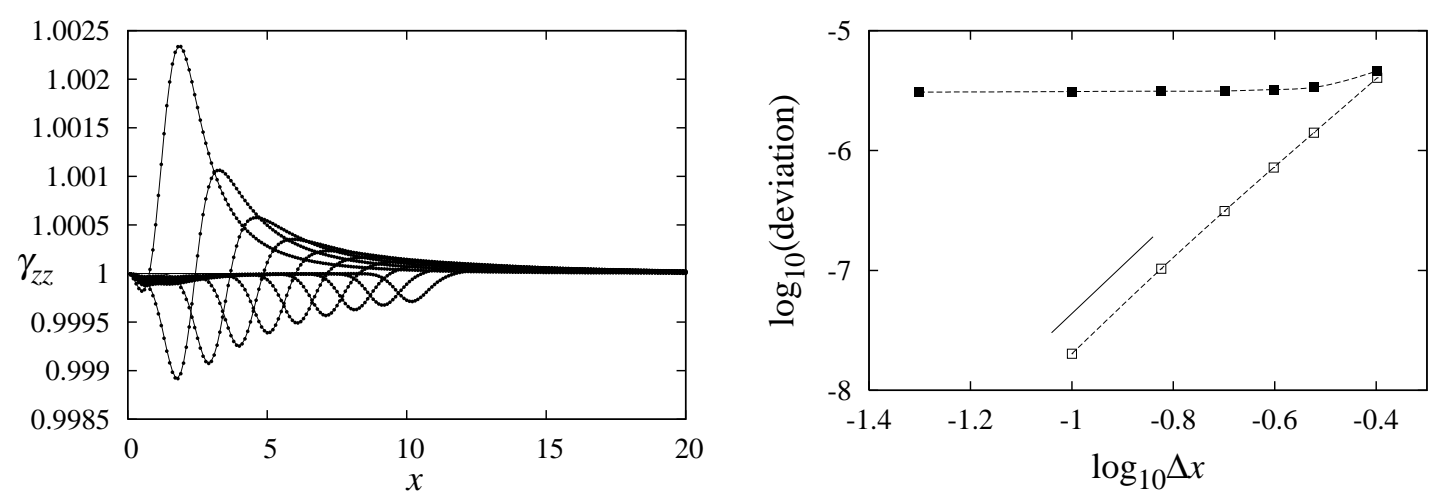

FIG. 6: Left-hand panel: Snapshots of $\gamma_{z z}$ along the $x$ axis for $t=1,2, \ldots, 10$ for propagation of a gravitational-wave packet. The dotted points $(\bullet)$ and solid curves denote the numerical results and perturbative solutions, respectively. Right-hand panel: The averaged deviation of $\gamma_{x x}$ from the analytic perturbative solution ( $\mathbf{\square})$ and that from the numerical data with the grid resolution $\Delta x=0.05(\square)$ as functions of $\Delta x$. Here, the data at time $t=3$ are used and the average is taken for the data in the range $0 \leq x \leq 6$ and $0 \leq z \leq 6$. The upper short line segment shows the relation of the fourth-order convergence (i.e., a segment with the slope 4).

and $\omega_{0}=2$ in Eq. (A23). In the simulation, we evolved the initial data that correspond to the perturbative solution under the same gauge condition $\alpha=1$ and $\beta^{i}=0$. The left-hand panel of Fig. 6] compares the analytic solution for the linearized Einstein equation (solid curves) and the numerical results (dotted points) obtained by the $2 \mathrm{D}$ " $x=y, z=w$ " code (where the double cartoon method is used). The values of $\gamma_{z z}$ are plotted along the $x$ axis for $t=1,2, \ldots, 10$. The two results agree well, indicating the validity of our code.

The right-hand panel of Fig. 6 shows the deviation of the numerical solution from the analytic solution of the perturbation as a function of grid sizes $\Delta x$ (black squares, $\mathbf{\square}$ ). Here, we used the data of $\gamma_{x x}$ on the $(x, z)$ plane at $t=3$ and evaluated the deviation by taking the average of $\left|\gamma_{x x}-\gamma_{x x}^{(a)}\right|$ in the region $0 \leq x \leq 6$ and $0 \leq z \leq 6$. The deviation scarcely depend on $\Delta x$, and thus it is not caused by the grid resolution. The deviation primarily comes from the fact that the perturbative solution ignores the second- and higher-order quantities in $h_{i j}$, whereas the numerical simulation is carried out by the fully nonlinear evolution equations. Indeed, the order of the difference $\sim 10^{-5}$ agrees with the magnitude of the nonlinear effect for our chosen wave amplitude.

Squares $(\square)$ in the same panel show the difference of the numerical data computed with 
the grid resolutions $\Delta x \geq 0.1$ from the one with the grid resolution $\Delta x=0.05$. The difference decreases approximately at the fourth order, implying that our numerical solution achieves the fourth-order convergence.

Using the analytic solution of the linearized Einstein equation, we can also test extraction methods of gravitational-wave energy flux from the numerical data. The Newman-Penrose variable is now widely used for extracting radiated energy of gravitational waves in the 4D numerical relativity. However, the formalism for the extraction based on the Weyl scalar has not been developed in higher dimensions. Thus, for the calculation of the energy flux, we adopt the Landau-Lifshitz pseudotensor $t_{\mathrm{LL}}^{\mu \nu}$ [40], whose $D$-dimensional form is given in Appendix B. Because $t_{\mathrm{LL}}^{\mu \nu}$ is not a tensor, it is a coordinate-dependent quantity in general. However, as discussed in p. 85 of Ref. [19], the total amount of gravitational energy and the total radiated energy, Eqs. (B8) and (Bי), are shown to be the gauge-invariant quantities for the linear gravitational waves of a perturbed flat spacetime up to second order with respect to the metric perturbation.

Let $r=r_{\text {obs }}$ be the radius of the extraction of gravitational-wave energy flux. The integrated energy flux $E_{\text {rad }}$ through the surface $r=r_{\text {obs }}$ is evaluated by

$$
E_{\mathrm{rad}}\left(r_{\mathrm{obs}}\right)=\int t_{\mathrm{LL}}^{0 r} d S d t
$$

where $d S$ is the area element of a hypersphere of $r=r_{\text {obs. }}$. Here, the second-order expression of the Landau-Lifshitz pseudotensor with respect to the perturbative quantities is used (see Eq. ( $(\overline{B 6})$ and subsequent explanation). We evaluate $E_{\text {rad }}\left(r_{\mathrm{obs}}\right)$ both for the perturbative solution and for the full numerical solution, and compare the two results.

The value of $E_{\text {rad }}\left(r_{\text {obs }}\right)$ for the perturbative solution is evaluated semianalytically by proceeding the integrations of Eq. (50) numerically. Figure 7 shows the value of $8 \pi G_{5} E_{\mathrm{rad}} \omega_{0}^{2}$ as a function of $r_{\text {obs }} \omega_{0}$ by the solid curve. $E_{\text {rad }}$ changes from zero to a constant value $\simeq 0.00925 / 8 \pi G_{5} \omega_{0}^{2}$ as $r_{\mathrm{obs}} \omega_{0}$ increases from zero to 40 . The value of $E_{\mathrm{rad}}$ near the center $r_{\mathrm{obs}} \omega_{0} \sim 1$ does not have a definite meaning because the Landau-Lifshitz pseudotensor is not gauge invariant. However, the asymptotic value of $E_{\mathrm{rad}}$ for $r_{\mathrm{obs}} \omega_{0} \gg 1$ is gauge invariant and should indicate the correct amount of the radiated energy. Note that because of the conservation law (B7), $\partial_{\mu} t_{\mathrm{LL}}^{\mu \nu}=0$, the integrated energy flux $E_{\mathrm{rad}}\left(r_{\mathrm{obs}}\right)$ has to be equal to the initial amount of energy within the surface $r=r_{\text {obs }}$,

$$
E\left(r_{\mathrm{obs}}\right)=\int_{r \leq r_{\mathrm{obs}}} t_{\mathrm{LL}}^{00} d V
$$




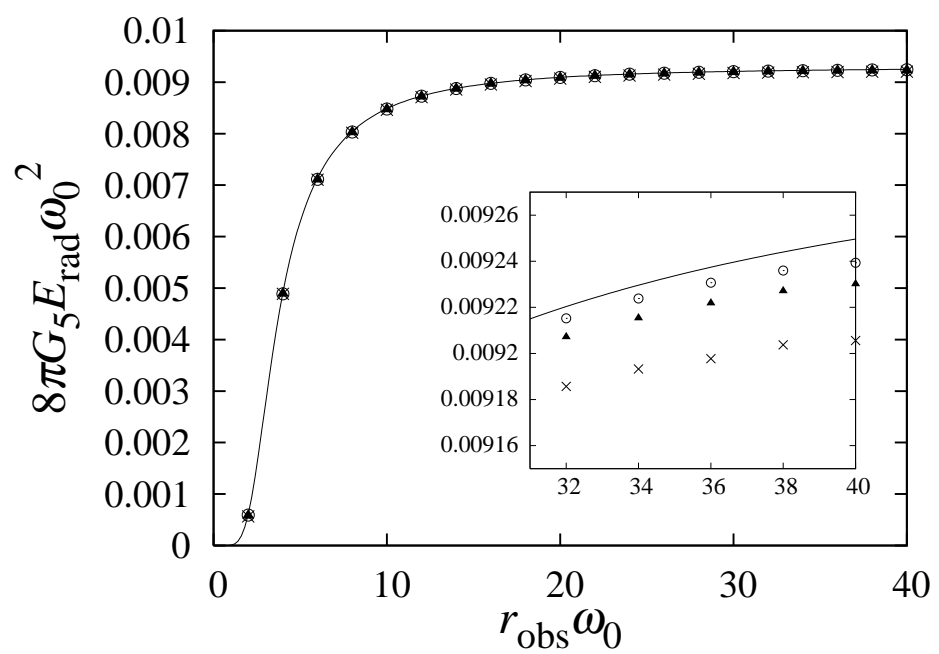

FIG. 7: The integrated energy flux $8 \pi G_{5} E_{\mathrm{rad}} \omega_{0}^{2}$ calculated by the Landau-Lifshitz pseudotensor as a function of the extraction radii $r_{\text {obs }} \omega_{0}$ for the analytic solution (solid curve) and for the numerical results computed with the grid resolutions $\Delta x=0.1(\odot), 0.15(\mathbf{\Lambda})$, and $0.2(\times)$. The inset shows the enlarged figure for the region $31 \leq r_{\mathrm{obs}} \omega_{0} \leq 40$.

where $d V$ is a volume element of the $4 \mathrm{D}$ space. This is directly checked by the numerical integrations.

In order to calculate $E_{\mathrm{rad}}\left(r_{\mathrm{obs}}\right)$ for the numerical solution, we proceed as follows: At each time step, we define the perturbed quantities as $h_{t t}=-2(\alpha-1), h_{t i}=\beta_{i}$, and $h_{i j}=\gamma_{i j}-\delta_{i j}$, and evaluate $t^{i 0}$ at $r=r_{\text {obs }}$ using Eq. (B6). Then, we calculate the integral

$$
\frac{d E_{\mathrm{rad}}}{d t}=\int t^{i 0} \hat{n}_{i} d S
$$

that gives the energy flux through a surface $r=r_{\text {obs }}$, where $\hat{n}_{i}$ is the outward unit normal to the surface. Finally, $d E_{\text {rad }} / d t$ is integrated from the initial time to the final time to obtain $E_{\text {rad }}\left(r_{\text {obs }}\right)$. In order to evaluate the metric functions for a surface of $r=r_{\text {obs }}$, we used linear, quadratic and cubic interpolations and compared the results. Although relatively large deviation from the analytic result is seen for the linear interpolation, the deviation becomes smaller when the quadratic interpolation is used. The result of the cubic interpolation did not improve that of the quadratic interpolation. This is because in these cases, the error primarily comes from the error generated at the outer boundary (i.e., the error due to the inaccuracy of the outgoing boundary condition) which is approximately at the second order with respect to the grid size. 
Figure 7 shows the results of $8 \pi G_{5} E_{\mathrm{rad}} \omega_{0}^{2}$ evaluated by the quadratic interpolation at several observation points for the grid resolutions $\Delta x=0.1(\odot), 0.15(\mathbf{\Delta})$, and $0.2(\times)$. The deviation decreases as the resolution is increased and the numerical data approach the analytic result.

We also evolved the gravitational-wave packet using the dynamical slicing and $\Gamma$-driver conditions and checked that $E_{\text {rad }}$ depends very weakly on the initial choice of the lapse and shift as long as the initial value of $\alpha-1$ and $\beta^{i}$ is small. This is natural because the gauge invariance of $E_{\text {rad }}$ is guaranteed for $r_{\text {obs }} \omega_{0} \gg 1$. Therefore, we conclude that the extraction of gravitational-wave energy flux by the Landau-Lifshitz pseudotensor works well, as far as the amplitude of gravitational waves is sufficiently small at the extracted region (i.e., in the wave zone).

\section{SUMMARY}

This paper describes the formulations for numerical relativity in higher dimensions and reports the new codes for simulating 5D spacetimes. We derived the BSSN formalism for higher-dimensional spacetimes and also studied the cartoon method in 5D spacetimes of $U(1)$ symmetry $(x=y, z, w), U(1) \times U(1)$ symmetry $(x=y, z=w)$, and $S O(3)$ symmetry $(x=$ $y=z, w)$. Based on the BSSN formalism and the cartoon methods, we have implemented the new 5D numerical relativity codes, and tested them by evolving the 5D Schwarzschild spacetime and the spacetime composed of gravitational waves of small amplitude. The numerical results converge to the analytic solutions with improving the grid resolution at the correct order (fourth order). It was also demonstrated that the 5D Schwarzschild spacetime can be evolved for a long time by the puncture approach, as in the $4 \mathrm{D}$ case.

We proposed the method of extracting gravitational-wave energy flux by the LandauLifshitz pseudotensor and tested this method. We showed that the integrated energy fluxes calculated at several surfaces $r=r_{\text {obs }}$ agree well with the semianalytic solution derived by perturbative calculations. Furthermore, it was confirmed that the result is insensitive to the gauge conditions for the lapse and shift. These results indicate that the energy extraction by the Landau-Lifshitz pseudotensor works well. The remaining problem would be to check that the extraction in the wave zone works well even when the central region is highly nonlinear, e.g., a head-on collision of two black holes. This will be tested by performing a simulation of 
Brill wave spacetime in five dimensions and by comparing the ADM mass of the initial data and the energy radiated during the evolution. Also, it is necessary to check if the extraction of the angular momentum is possible. We expect that the radiated angular momentum also can be calculated by the Landau-Lifshitz pseudotensor using a similar manner to the 4D case [48].

As discussed in Sec. I, there are many interesting issues of nonlinear dynamics in higherdimensional gravity, which should be studied in numerical relativity. In this paper, we have prepared the tools necessary for simulating higher-dimensional spacetimes. Our next step is to apply our codes to the unsolved problems.

\section{Acknowledgments}

We thank K. Nakao, T. Shiromizu, and T. Tanaka for discussions, and HY also thanks V. Cardoso for comments. Numerical computations were in part performed on the NEC-SX9 at CfCA in National Astronomical Observatory of Japan and on the NEC-SX8 at Yukawa Institute for Theoretical Physics (YITP) at Kyoto University. HY thanks YITP, where this work was initiated, for financial support and hospitality. MS was in part supported by Grant-in-Aid for Scientific Research (21340051) and by Grant-in-Aid for Scientific Research on Innovative Area (20105004) of the Japanese Monbukagakusho.

\section{APPENDIX A: LINEAR GRAVITATIONAL WAVES IN THE 5D SPACETIME}

In this section, we describe solutions of the linearized Einstein equations in the 5D flat spacetime focusing on the perturbations preserving $U(1) \times U(1)$ symmetry and $S O(3)$ symmetry. In the following, we denote the metric perturbation as $h_{\mu \nu}$, which obeys the linearized Einstein equation

$$
\delta G_{\mu \nu}\left[h_{\alpha \beta}\right]=0
$$

The analysis for perturbations of higher-dimensional Schwarzschild black holes described in Ref. [49] is partially used, since this formulation is applicable also for the flat spacetime. In their approach, the perturbation is decomposed into the scalar, vector, and tensor modes (with respect to the 3D unit sphere) using spherical harmonic functions, and the master equations are derived for the gauge-invariant variables. We adopt their method for spherical 
harmonic expansion but do not use the master equations, because we are interested in explicit special solutions for which the master equations are not necessary.

\section{Perturbation with $U(1) \times U(1)$ symmetry}

We here derive a solution of $U(1) \times U(1)$ symmetry for Eq. (A1). Denoting the coordinates by $(t, x, y, z, w)$, we introduce the following curvilinear coordinates:

$$
\begin{aligned}
& x=r \sin \theta \cos \varphi, \\
& y=r \sin \theta \sin \varphi, \\
& z=r \cos \theta \cos \psi, \\
& w=r \cos \theta \sin \psi .
\end{aligned}
$$

In these coordinates, the line element of the flat space is

$$
d l^{2}=d r^{2}+r^{2}\left(d \theta^{2}+\sin ^{2} \theta d \varphi^{2}+\cos ^{2} \theta d \psi^{2}\right) .
$$

For the $U(1) \times U(1)$ symmetry case, the linear perturbation $h_{\mu \nu}$ satisfies

$$
\frac{\partial h_{\mu \nu}}{\partial \varphi}=\frac{\partial h_{\mu \nu}}{\partial \psi}=0
$$

since $\partial_{\varphi}$ and $\partial_{\psi}$ are the Killing vectors.

In the gauge condition with $\alpha=1$ and $\beta^{k}=0$ (i.e., $h_{00}=h_{0 i}=0$ ), the spatial components of the linear perturbation $h_{i j}$ satisfy

$$
\ddot{h}_{i j}=\Delta h_{i j}
$$

where $\Delta$ is the flat $4 \mathrm{D}$ Laplacian. The Hamiltonian and momentum constraints in the linear approximation give

$$
\begin{aligned}
& \bar{g}^{i j} h_{i j}=0, \\
& \bar{D}^{i} h_{i j}=a_{j},
\end{aligned}
$$

where $\bar{g}_{i j}$ is the $4 \mathrm{D}$ flat space metric in the curvilinear coordinates and $\bar{D}_{i}$ is the covariant derivative with respect to $\bar{g}_{i j}$. $a_{j}$ denotes a constant vector determined at the initial state, which is set to be zero in the following for simplicity. 


\section{a. Scalar mode}

The scalar mode (with respect to a 3D unit sphere) is expanded in terms of scalar harmonic functions $\mathbb{S}$ on a $3 \mathrm{D}$ unit sphere with the metric $d \sigma^{2}=\sigma_{I J} d z^{I} d z^{J}=d \theta^{2}+\sin ^{2} \theta d \varphi^{2}+$ $\cos ^{2} \theta d \psi^{2}$, which satisfy the equation

$$
\left[\hat{\Delta}_{3}+l(l+2)\right] \mathbb{S}=0
$$

where $\hat{\Delta}_{3}=\hat{D}^{I} \hat{D}_{I}$ is the Laplace operator on the 3D unit sphere. In the following, we focus only on solutions of the lowest-order multipole moment $l=2$, for which the harmonic function is

$$
\mathbb{S}=2 \cos ^{2} \theta-1
$$

The scalar-mode perturbation is given in the form

$$
h_{i j}=\left(\begin{array}{cc}
a(t, r) \mathbb{S} & r b(t, r) \mathbb{S}_{J} \\
* & r^{2}\left[c(t, r) \mathbb{S} \sigma_{I J}+d(t, r) \mathbb{S}_{I J}\right]
\end{array}\right)
$$

where

$$
\mathbb{S}_{J}:=\hat{D}_{J} \mathbb{S} ; \quad \text { and } \quad \mathbb{S}_{I J}:=\frac{1}{l(l+2)} \hat{D}_{I} \hat{D}_{J} \mathbb{S}+\frac{1}{3} \sigma_{I J} \mathbb{S},
$$

or more explicitly,

$$
\mathbb{S}_{I J}=\frac{1}{6} \times\left(\begin{array}{ccc}
1-2 \cos ^{2} \theta & 0 & 0 \\
* & \sin ^{2} \theta\left(\cos ^{2} \theta-2\right) & 0 \\
* & * & \cos ^{2} \theta\left(1+\cos ^{2} \theta\right)
\end{array}\right) .
$$

Equation (A9) gives $a+3 c=0$, and Eq. (A10) yields

$$
\begin{gathered}
8 b=4 a+r a_{, r}, \\
\frac{5}{12} d=4 b+r b_{, r}+c .
\end{gathered}
$$

The $r r$ component of Eq. (A8) with Eq. (A17) gives a wave equation for $a$ :

$$
\ddot{a}=a_{, r r}+\frac{7}{r} a_{, r} .
$$

Equations (A17) and (A18) imply that once $a$ is computed from the equation (A19), $b, c$ and $d$ are subsequently determined. The obtained solution is guaranteed to satisfy other components of Eq. (A8). 
Defining $a \equiv u / r^{3}$, we obtain the equation

$$
\ddot{u}=u_{, r r}+\frac{1}{r} u_{, r}-\frac{n^{2}}{r^{2}} u .
$$

Here, $n=l+1=3$. The formal solution of this equation is written as

$$
u=\operatorname{Re}\left[\int d \omega f(\omega) e^{i \omega t} J_{n}(\omega r)\right]
$$

where $f(\omega)$ is an arbitrary function of $\omega$, and $J_{n}$ is the Bessel function of $n$-th order. In the integral expression, it is written by

$$
J_{n}(z)=\frac{1}{2 \pi} \int_{0}^{2 \pi} d \vartheta \cos (n \vartheta-z \sin \vartheta)
$$

To constitute a solution for the propagation of a gravitational-wave packet, we set $f(\omega)=$ $-i \sqrt{2 \pi} A_{0} e^{-\omega^{2} / 2 \omega_{0}^{2}}$. Then, Eq. (A21) is integrated to give

$$
u(t, r)=A_{0} \omega_{0} \int_{0}^{2 \pi} d \vartheta \sin (n \vartheta) e^{-\omega_{0}^{2}(t-r \sin \vartheta)^{2} / 2} .
$$

In this case, $u=0$ at $t=0$, and thus $h_{i j}=0$, whereas the extrinsic curvature $K_{i j}=-\dot{h}_{i j} / 2$ is not zero because

$$
\dot{u}(0, r)=r A_{0} \omega_{0}^{3} \int_{0}^{2 \pi} d \vartheta \sin (n \vartheta) \sin \vartheta e^{-\left(\omega_{0} r \sin \vartheta\right)^{2} / 2}
$$

is not zero at $t=0$.

\section{b. Vector mode}

Perturbation $h_{i j}$ of the vector type is expanded in terms of the harmonic vectors $\mathbb{V}_{I}$ satisfying

$$
\begin{gathered}
{\left[\hat{\Delta}_{3}+l(l+2)-1\right] \mathbb{V}_{I}=0} \\
\hat{D}_{J} \mathbb{V}^{J}=0 .
\end{gathered}
$$

Under the $U(1) \times U(1)$ symmetry, only the modes for odd $l$ numbers are nonzero. Since the $l=1$ mode denotes a stationary perturbation with angular momentum, the lowest value of $l$ is 3 for the nonstationary perturbation. For this mode,

$$
\mathbb{V}^{J}=\left(0, A\left(\sin ^{2} \theta-2 / 3\right), B\left(\cos ^{2} \theta-2 / 3\right)\right)
$$


where $A$ and $B$ are arbitrary constants. The perturbation is given in the form

$$
h_{i j}=\left(\begin{array}{cc}
0 & (1 / r) k(t, r) \mathbb{V}_{J} \\
* & r o(t, r) \mathbb{V}_{I J}
\end{array}\right),
$$

where $\mathbb{V}_{I J}$ is defined by

$$
\begin{aligned}
\mathbb{V}_{I J}: & =\frac{1}{2}\left(\hat{D}_{I} \mathbb{V}_{J}+\hat{D}_{J} \mathbb{V}_{I}\right) \\
& =\left(\begin{array}{ccc}
0 & A \sin ^{3} \theta \cos \theta & -B \sin \theta \cos ^{3} \theta \\
* & 0 & 0 \\
* & * & 0
\end{array}\right) .
\end{aligned}
$$

From Eqs. (A8) and (A10), the equations for $k$ and $o$ are derived as

$$
\begin{gathered}
\ddot{k}=k_{, r r}+\frac{1}{r} k_{, r}-\frac{16}{r^{2}} k, \\
6 o=k_{, r}+\frac{2}{r} k .
\end{gathered}
$$

Here, Eq. (A30) has the same form as Eq. (A20) but with $n=4$. Hence, a special solution for $k(t, r)$ is given by Eq. (A23) with $n=4$, and then $o(t, r)$ is calculated from Eq. (A31).

\section{c. Tensor mode}

Perturbation $h_{i j}$ of the tensor type is expanded in terms of the harmonic tensors $\mathbb{T}_{I J}$ satisfying

$$
\begin{gathered}
{\left[\hat{\Delta}_{3}+l(l+2)-2\right] \mathbb{T}_{I J}=0} \\
\mathbb{T}_{I}^{I}=0, \quad \hat{D}_{J} \mathbb{T}^{J}=0 .
\end{gathered}
$$

Under $U(1) \times U(1)$ symmetry, the possible harmonic tensors for $l=2$ are

$$
\mathbb{T}_{I J}=\left(\begin{array}{ccc}
A & 0 & 0 \\
* & A \sin ^{2} \theta\left(1-3 \sin ^{2} \theta\right) & B \sin ^{2} \theta \cos ^{2} \theta \\
* & * & A \cos ^{2} \theta\left(3 \sin ^{2} \theta-2\right)
\end{array}\right),
$$

where $A$ and $B$ are arbitrary constants. The perturbation is given in the form

$$
h_{i j}=\left(\begin{array}{cc}
0 & 0 \\
* & r h(t, r) \mathbb{T}_{I J}
\end{array}\right),
$$


and from Eq. (A8), the equation for $h$ becomes

$$
\ddot{h}=h_{, r r}+\frac{1}{r} h_{, r}-\frac{9}{r^{2}} h .
$$

This is the same equation as Eq. (A20), and thus a special solution is given by Eq. (A23) with $n=3$.

\section{Perturbation with $S O(3)$ symmetry}

Next, we derive solutions of a perturbation of $S O(3)$ symmetry. For the Minkowski coordinates $(t, x, y, z, w)$, we introduce the following hyperspherical coordinates:

$$
\begin{aligned}
& x=r \sin \theta \sin \varphi \sin \psi, \\
& y=r \sin \theta \sin \varphi \cos \psi, \\
& z=r \sin \theta \cos \varphi, \\
& w=r \cos \theta .
\end{aligned}
$$

Then, the line element of the flat space is given by

$$
d l^{2}=d r^{2}+r^{2}\left(d \theta^{2}+\sin ^{2} \theta d \varphi^{2}+\sin ^{2} \theta \sin ^{2} \varphi d \psi^{2}\right) .
$$

Here, we consider solutions of $S O(3)$ symmetry with the Killing vectors

$$
\begin{aligned}
& \boldsymbol{\xi}_{1}=-\cos \psi \partial_{\varphi}+\cot \varphi \sin \psi \partial_{\psi}, \\
& \boldsymbol{\xi}_{2}=\sin \psi \partial_{\varphi}+\cot \varphi \cos \psi \partial_{\psi}, \\
& \boldsymbol{\xi}_{3}=\partial_{\psi}
\end{aligned}
$$

Under the requirement of this symmetry, the vector and tensor modes do not exist, because there are no vector and tensor harmonic functions that satisfy $\mathcal{L}_{\xi_{n}} \mathbb{V}_{I}=0$ and $\mathcal{L}_{\xi_{n}} \mathbb{T}_{I J}=0$. Therefore, only the scalar mode should be analyzed.

The scalar harmonic function defined by Eq. (A11) on a 3D unit sphere with the metric $d \sigma^{2}=\sigma_{I J} d z^{I} d z^{J}=d \theta^{2}+\sin ^{2} \theta d \varphi^{2}+\sin ^{2} \theta \sin ^{2} \varphi d \psi^{2}$ is

$$
\mathbb{S}=4 \cos ^{2} \theta-1, \quad \text { for } \quad l=2 .
$$

The metric perturbation is given in the same form as Eq. (A13). Here, definitions for $\mathbb{S}_{I}$ and $\mathbb{S}_{I J}$ are same as Eq. (A14), and their explicit forms in this case are

$$
\mathbb{S}_{J}=(-8 \sin \theta \cos \theta, 0,0),
$$




$$
\mathbb{S}_{I J}=\frac{1}{3} \sin ^{2} \theta \times\left(\begin{array}{ccc}
2 & 0 & 0 \\
* & -\sin ^{2} \theta & 0 \\
* & * & -\sin ^{2} \theta \sin ^{2} \varphi
\end{array}\right) .
$$

The equations for $a, b, c$, and $d$ are the same as Eqs. (A17)-(A19), and a special solution for $u=r^{3} a$ is given by the same formula as Eq. (A23).

\section{APPENDIX B: LANDAU-LIFSHITZ PSEUDOTENSOR}

In this section, we derive the Landau-Lifshitz pseudotensor in a $D$-dimensional spacetime $\mathcal{M}$ with the metric $g_{\mu \nu}$. Following [40], we define

$$
\tilde{g}^{\mu \nu}=\sqrt{-g} g^{\mu \nu}
$$

where $g$ is the determinant of the metric, and then we introduce the super-potential

$$
H^{\mu \alpha \nu \beta}=\tilde{g}^{\mu \nu} \tilde{g}^{\alpha \beta}-\tilde{g}^{\alpha \nu} \tilde{g}^{\mu \beta}
$$

The Landau-Lifshitz pseudotensor is defined by

$$
16 \pi G_{D} t_{\mathrm{LL}}^{\mu \nu}=(-g)^{-1} H_{, \alpha \beta}^{\mu \alpha \nu \beta}-\left(2 R^{\mu \nu}-g^{\mu \nu} R\right) .
$$

From this definition, the conservation law is derived:

$$
\left[(-g)\left(T^{\mu \nu}+t_{\mathrm{LL}}^{\mu \nu}\right)\right]_{, \nu}=0
$$

Because the Landau-Lifshitz pseudotensor is not a tensor, it does not have a covariant meaning in general. However, for a perturbed flat spacetime, the leading-order terms of $t_{\mathrm{LL}}^{\mu \nu}$ with respect to the perturbative quantities can be used to evaluate the total energy and total radiated energy of the gravitational field in a gauge-invariant manner (see below).

In Ref. [40], two expressions for $t_{\mathrm{LL}}^{\mu \nu}$ are given. The first one is the expression in terms of the Christoffel symbols, and this expression holds for arbitrary dimensionality $D$. The second one is the expression by the metric functions, and it is modified to give

$$
\begin{aligned}
16 \pi G_{D}(-g) t_{\mathrm{LL}}^{\mu \nu}= & \tilde{g}_{, \alpha}^{\mu \nu} \tilde{g}_{, \beta}^{\alpha \beta}-\tilde{g}_{, \alpha}^{\mu \alpha} \tilde{g}_{, \beta}^{\nu \beta}+\frac{1}{2} g^{\mu \nu} g_{\alpha \beta} \tilde{g}_{, \sigma}^{\alpha \rho} \tilde{g}_{, \rho}^{\sigma \beta} \\
& -\left(g^{\mu \alpha} g_{\beta \rho} \tilde{g}_{, \sigma}^{\nu \rho} \tilde{g}_{, \alpha}^{\beta \sigma}+g^{\nu \alpha} g_{\beta \rho} \tilde{g}_{, \sigma}^{\mu \rho} \tilde{g}_{, \alpha}^{\beta \sigma}\right)+g_{\alpha \beta} g^{\rho \sigma} \tilde{g}_{, \rho}^{\mu \alpha} \tilde{g}_{, \sigma}^{\nu \beta} \\
& +\frac{1}{4(D-2)}\left(2 g^{\mu \alpha} g^{\nu \beta}-g^{\mu \nu} g^{\alpha \beta}\right)\left[(D-2) g_{\rho \sigma} g_{\gamma \delta}-g_{\sigma \gamma} g_{\rho \delta}\right] \tilde{g}_{, \alpha}^{\rho \delta} \tilde{g}_{, \beta}^{\sigma \gamma}
\end{aligned}
$$


in $D$ dimensions. Let us consider the perturbation on a flat spacetime, whose metric is $g_{\mu \nu}=\eta_{\mu \nu}+h_{\mu \nu}$, where $\eta_{\mu \nu}$ is the flat metric in the Minkowski coordinates. Defining $\hat{h}_{\mu \nu}:=$ $h_{\mu \nu}-(1 / 2) h \eta_{\mu \nu}$, the Landau-Lifshitz pseudotensor is rewritten as

$$
\begin{aligned}
16 \pi G_{D} t_{\mathrm{LL}}^{\mu \nu}=\hat{h}_{, \alpha}^{\mu \nu} \hat{h}_{, \beta}^{\alpha \beta}-\hat{h}_{, \alpha}^{\mu \alpha} \hat{h}_{, \beta}^{\nu \beta}+\frac{1}{2} \eta^{\mu \nu} \hat{h}_{, \sigma}^{\alpha \rho} \hat{h}_{\alpha, \rho}^{\sigma} & \\
& -\left(\hat{h}_{, \sigma}^{\mu \rho} \hat{h}_{\rho}^{\sigma, \nu}+\hat{h}_{, \sigma}^{\nu \rho} \hat{h}_{\rho}^{\sigma, \mu}\right)+\hat{h}^{\mu \alpha, \rho} \hat{h}_{\alpha, \rho}^{\nu} \\
+ & \frac{1}{2} \hat{h}^{\rho \sigma, \mu} \hat{h}_{\rho \sigma}{ }^{\nu}{ }^{\nu}-\frac{1}{4} \eta^{\mu \nu} \hat{h}^{\rho \sigma, \alpha} \hat{h}_{\rho \sigma, \alpha}-\frac{1}{4(D-2)}\left(2 \hat{h}^{, \mu} \hat{h}^{, \nu}-\eta^{\mu \nu} \hat{h}^{, \alpha} \hat{h}_{, \alpha}\right) .
\end{aligned}
$$

Here, we have kept only the second-order quantities of the perturbation. Note that the second-order Landau-Lifshitz pseudotensor $t_{\mathrm{LL}}^{\mu \nu}$ behaves as a tensor against the general coordinate transformations of the background spacetime (but for a fixed gauge), by replacing the coordinate derivatives $(, \mu)$ to the covariant derivatives and the Minkowski metric $\eta^{\mu \nu}$ to the flat background metric $\bar{g}^{\mu \nu}$ in curved coordinates in Eq. (B6). The quantity $t^{0 r}$ in Eq. (50) has to be evaluated in this way.

For the expression ( $(\mathrm{B} 6)$, the conservation law (B4) for a vacuum spacetime becomes

$$
\partial_{\mu} t_{\mathrm{LL}}^{\mu \nu}=0
$$

in the Minkowski coordinates, which suggests that $t_{\mathrm{LL}}^{\mu \nu}$ can be interpreted as the effective stress-energy tensor of the gravitational field valid up to second order in $h_{\mu \nu}$. Here, we have to be careful because the Landau-Lifshitz pseudotensor is not the unique quantity satisfying the condition (B7) and also because this quantity is not gauge invariant (see Ref. [19]). However, the total energy

$$
E_{\mathrm{tot}}=\int t_{\mathrm{LL}}^{00} d V
$$

is shown to be the gauge-invariant quantity, where $d V$ is the volume element of the spacelike hypersurface. Similarly, the total radiated energy

$$
E_{\mathrm{rad}}=\int t^{0 i} \hat{n}_{i} d S d t
$$

is gauge-invariant, where $d S$ and $\hat{n}^{i}$ are the area element and an outward unit normal of a surface at the distant region. Therefore, the Landau-Lifshitz pseudotensor $t_{\text {LL }}^{\mu \nu}$ provides us 
a reliable method for evaluating the total radiated energy.

[1] N. Arkani-Hamed, S. Dimopoulos and G. R. Dvali, Phys. Lett. B 429, 263 (1998) arXiv:hep-ph/9803315;

I. Antoniadis, N. Arkani-Hamed, S. Dimopoulos and G. R. Dvali, ibid. 436, 257 (1998) arXiv:hep-ph/9804398.

[2] L. Randall and R. Sundrum, Phys. Rev. Lett. 83, 3370 (1999) arXiv:hep-ph/9905221;

L. Randall and R. Sundrum, ibid. 83, 4690 (1999) arXiv:hep-th/9906064].

[3] T. Banks and W. Fischler, arXiv:hep-th/9906038.

[4] S. Dimopoulos and G. Landsberg, Phys. Rev. Lett. 87, 161602 (2001) arXiv:hep-ph/0106295.

[5] S. B. Giddings and S. Thomas, Phys. Rev. D 65, 056010 (2002) arXiv:hep-ph/0106219.

[6] P. Kanti, Lect. Notes Phys. 769, 387 (2009) arXiv:0802.2218 [hep-th]].

[7] F. Pretorius, Phys. Rev. Lett. 95, 121101 (2005) arXiv:gr-qc/0507014.

[8] M. Campanelli, C. O. Lousto, P. Marronetti and Y. Zlochower, Phys. Rev. Lett. 96, 111101 (2006) arXiv:gr-qc/0511048.

[9] J. G. Baker, J. Centrella, D. I. Choi, M. Koppitz and J. van Meter, Phys. Rev. Lett. 96, 111102 (2006) arXiv:gr-qc/0511103.

[10] P. Diener et al., Phys. Rev. Lett. 96, 121101 (2006) arXiv:gr-qc/0512108.

[11] F. Herrmann, D. Shoemaker and P. Laguna, arXiv:gr-qc/0601026.

[12] M. Boyle, D. A. Brown, L. E. Kidder, A. H. Mroue, H. P. Pfeiffer, M. A. Scheel, G. B. Cook, and S. A. Teukolsky, Phys. Rev. D 76, 124038 (2007) arXiv:0710.0158 [gr-qc]].

[13] M. Choptuik, L. Lehner, I. I. Olabarrieta, R. Petryk, F. Pretorius and H. Villegas, Phys. Rev. D 68, 044001 (2003) arXiv:gr-qc/0304085.

[14] D. Garfinkle, L. Lehner and F. Pretorius, Phys. Rev. D 71, 064009 (2005) arXiv:gr-qc/0412014.

[15] H. Yoshino and Y. Nambu, Phys. Rev. D 67, 024009 (2003) arXiv:gr-qc/0209003.

[16] H. Yoshino and V. S. Rychkov, Phys. Rev. D 71, 104028 (2005) arXiv:hep-th/0503171.

[17] D. M. Eardley and S. B. Giddings, Phys. Rev. D 66, 044011 (2002), arXiv:gr-qc/0201034.

[18] P. C. Aichelburg and R. U. Sexl, Gen. Rel. Grav. 2, 303 (1971).

[19] R. Wald, General Relativity (The University of Chicago Press, Chicago, 1984). 
[20] U. Sperhake, V. Cardoso, F. Pretorius, E. Berti and J. A. González, Phys. Rev. Lett. 101, 161101 (2008) arXiv:0806.1738 [gr-qc]].

[21] M. Shibata, H. Okawa and T. Yamamoto, Phys. Rev. D 78, 101501(R) (2008) arXiv:0810.4735 $[\mathrm{gr}-\mathrm{qc}]]$.

[22] U. Sperhake, V. Cardoso, F. Pretorius, E. Berti, T. Hinderer and N. Yunes, arXiv:0907.1252 $[\mathrm{gr}-\mathrm{qc}]$.

[23] R. C. Myers and M. J. Perry, Annals Phys. 172, 304 (1986).

[24] K. Murata and J. Soda, Prog. Theor. Phys. 120, 561 (2008) arXiv:0803.1371 [hep-th]].

[25] T. Oota and Y. Yasui, arXiv:0812.1623 [hep-th].

[26] O. J. C. Dias, P. Figueras, R. Monteiro, J. E. Santos and R. Emparan, arXiv:0907.2248 [hep-th].

[27] R. Emparan and R. C. Myers, JHEP 0309, 025 (2003) arXiv:hep-th/0308056.

[28] H. Yoshino, JHEP 0901, 068 (2009) [arXiv:0812.0465 [gr-qc]].

[29] H. Kudoh, T. Tanaka and T. Nakamura, Phys. Rev. D 68, 024035 (2003) arXiv:gr-qc/0301089.

[30] T. Tanaka, Prog. Theor. Phys. Suppl. 148, 307 (2003) arXiv:gr-qc/0203082.

[31] R. Emparan, A. Fabbri and N. Kaloper, JHEP 0208, 043 (2002) arXiv:hep-th/0206155.

[32] M. Shibata and T. Nakamura, Phys. Rev. D 52, 5428 (1995).

[33] T. W. Baumgarte and S. L. Shapiro, Phys. Rev. D 59, 024007 (1998) arXiv:gr-qc/9810065.

[34] M. Alcubierre, S. Brandt, B. Brügmann, D. Holz, E. Seidel, R. Takahashi and J. Thornburg, Int. J. Mod. Phys. D 10, 273 (2001) arXiv:gr-qc/9908012.

[35] M. Shibata, Prog. Theor. Phys. 104, 325 (2000) arXiv:gr-qc/0007049.

[36] M. Shibata, Phys. Rev. D 67, 024033 (2003) arXiv:gr-qc/0301103.

[37] B. Brügmann, J. A. González, M. Hannam, S. Husa, U. Sperhake and W. Tichy, Phys. Rev. D 77, 024027 (2008) arXiv:gr-qc/0610128.

[38] T. Yamamoto, M. Shibata and K. Taniguchi, Phys. Rev. D 78, 064054 (2008) arXiv:0806.4007 [gr-qc]].

[39] M. Alcubierre, B. Brügmann, P. Diener, M. Koppitz, D. Pollney, E. Seidel and R. Takahashi, Phys. Rev. D 67, 084023 (2003) arXiv:gr-qc/0206072.

[40] L. D. Landau and E. M. Lifshitz, The Classical Theory of Fields (Pergamon Press, Oxford, 1962). 
[41] M. Hannam, S. Husa, D. Pollney, B. Brügmann and N. Ó Murchadha, Phys. Rev. Lett. 99, 241102 (2007) arXiv:gr-qc/0606099].

[42] M. Hannam, S. Husa, B. Brügmann, J. A. González, U. Sperhake and N. Ó. Murchadha, J. Phys. Conf. Ser. 66, 012047 (2007) arXiv:gr-qc/0612097.

[43] I. Novikov, Ph.D. thesis, Shternberg Astronomical Institute, Moscow, 1963.

[44] C. Misner, K. Thorne, and J. Wheeler, Gravitation (W. H. Freeman and Company, San Francisco, 1973), pp. 826.

[45] F. Estabrook, H. Wahlquist, S. Christensen, B. DeWitt, L. Smarr and E. Tsiang, Phys. Rev. D 7, 2814 (1973).

[46] T. W. Baumgarte and S. G. Naculich, Phys. Rev. D 75, 067502 (2007) arXiv:gr-qc/0701037.

[47] K.-i. Nakao, H. Abe, H. Yoshino and M. Shibata, in progress.

[48] K. S. Thorne, Rev. Mod. Phys. 52, 299 (1980).

[49] H. Kodama and A. Ishibashi, Prog. Theor. Phys. 110, 701 (2003) arXiv:hep-th/0305147]. 